Pacific

Journal of

Mathematics

ON EXISTENCE OF A CLASSICAL SOLUTION TO

A GENERALIZED KELVIN-VOIGT MODEL

Miroslav BulíčeK, Petr KaPlickÝ AND Mark Steinhauer 


\title{
ON EXISTENCE OF A CLASSICAL SOLUTION TO A GENERALIZED KELVIN-VOIGT MODEL
}

\author{
Miroslav Bulíček, Petr KaplickÝ and Mark Steinhauer
}

\begin{abstract}
We consider a two-dimensional generalized Kelvin-Voigt model describing a motion of a compressible viscoelastic body. We establish the existence of a unique classical solution to such a model in the spatially periodic setting. The proof is based on Meyers' higher integrability estimates that guarantee the Hölder continuity of the gradient of velocity and displacement.
\end{abstract}

\section{Introduction}

In this paper we focus on qualitative properties of a solution to a generalized Kelvin-Voigt model that describes the motion of a two-dimensional compressible viscoelastic body. Hence, assuming that the body occupies a domain $\Omega:=(0,1)^{2}$ and that $T>0$ is the length of time interest, such a model is described by the system of equations

$$
\begin{aligned}
\rho_{0} \boldsymbol{u}_{t t}-\operatorname{div} \mathbf{T} & =\rho_{0} \boldsymbol{f} & & \text { in } Q, \\
\boldsymbol{u}(0, \cdot) & =\boldsymbol{u}_{0}(\cdot) & & \text { in } \Omega, \\
\boldsymbol{u}_{t}(0, \cdot) & =\boldsymbol{v}_{0}(\cdot) & & \text { in } \Omega,
\end{aligned}
$$

where $Q:=(0, T) \times \Omega$. Here, $\rho_{0}: \Omega \rightarrow \mathbb{R}_{+}$is a given density of the body, assumed to be time-independent, $f: Q \rightarrow \mathbb{R}^{2}$ is a given density of external body forces, $\boldsymbol{u}: Q \rightarrow \mathbb{R}^{2}$ denotes an unknown displacement field and $\mathbf{T}: Q \rightarrow \mathbb{R}^{2 \times 2}$ stands for the Cauchy stress tensor. The initial displacement is denoted by $\boldsymbol{u}_{0}: \Omega \rightarrow \mathbb{R}^{2}$ and the initial velocity of the body is $\boldsymbol{v}_{0}: \Omega \rightarrow \mathbb{R}^{2}$.

We assume that

$$
\mathbf{T}=\mathbf{T}^{T} \quad \text { in } Q
$$

Bulíček is a researcher of the University Center for Mathematical Modeling, Applied Analysis and Computational Mathematics (Math MAC). Kaplický is supported by grant 201/09/0917 of CSF, and also partially by the research project MSM 0021620839 financed by MEYS. Steinhauer acknowledges the support of the Nečas Center for Mathematical Modeling, project LC06052, and thanks the Center for its hospitality.

MSC2000: 35B65, 35Q74, 74D10.

Keywords: Kelvin-Voigt model, regularity, classical solution, large-data and long-time. 
and that $\mathbf{T}$ is given as a sum of a viscous and an elastic part,

$$
\begin{aligned}
\mathbf{T} & =\mathbf{T}_{v}+\mathbf{T}_{e}, \\
\mathbf{T}_{e} & =\mathbf{H}(\mathbf{D}(\boldsymbol{u})), \\
\mathbf{T}_{v} & =\mathbf{G}\left(\mathbf{D}\left(\boldsymbol{u}_{t}\right)\right),
\end{aligned}
$$

where $\mathbf{H}, \mathbf{G}: \mathbb{R}_{\mathrm{sym}}^{2 \times 2} \rightarrow \mathbb{R}_{\mathrm{sym}}^{2 \times 2}$ are continuous mappings and $\mathbf{D}=\left(\nabla+\nabla^{T}\right) / 2$ is the symmetric part of the gradient.

In the context of continuum mechanics, (1-1) 1 represents the balance of linear momentum written in Lagrangian coordinates. The decomposition (1-3) of the Cauchy stress tensor corresponds to the fact that the material under consideration is compressible. The initial density of the body is the given function $\rho_{0}$, while the density at time $t>0$ can be reconstructed from a formula $\rho(t, \cdot)(1+\operatorname{div} \boldsymbol{u}(t, \cdot))=\rho_{0}$; see [Bulíček et al. 2012, (26)]. Note that using the balance of angular momentum, the natural requirement for nonpolar materials is (1-2).

In general, most materials can be understood as viscoelastic and one can try to investigate their properties in full generality. Unfortunately, the resulting system is highly nonlinear and may be even hyperbolic and up to our best knowledge there is no satisfactory existence theory for such problems. Therefore it seems to be reasonable (and also necessary) to simplify the model in such a way that it still captures all essential phenomena but it is easier to handle from the mathematical (and even computational) point of view. One such possible procedure, which is also used here, is the assumption that the strains are small. Then, following the fundamental works of Kelvin [Thomson 1865] and Voigt [1892] and taking G and $\mathbf{H}$ to be linear operators, one obtains the standard Kelvin-Voigt model for a viscoelastic body. However, doing such simplification, and recalling that at the beginning we assumed that the strains were small, we directly obtained a model, where also stresses must be small. On the other hand, it is not true in the original model that even under the assumption that strains are small the Cauchy stress cannot be large, which is the main drawback of the linear Kelvin-Voigt model. Therefore, recently Rajagopal [2009] has reconsidered generalizations of the classical KelvinVoigt model wherein he allowed for both the elastic solid and viscous fluid to be described through implicit constitutive relations. These models were also obtained by considering small strains, but the essential assumption was that the strain is a function of the stress. Then using a linearization procedure, one can still end up with small deformations but keeps the essential nonlinearity in stresses. For a more sophisticated discussion, we refer the interested reader to [Rajagopal 2009] and [Bulíček et al. 2012], where the elastic and viscous part of the Cauchy stress are given by the general formula (1-4)-(1-5). This is also the model we are interested in here and one can think of the bodies described by these models as of mixtures 
of a material that can store energy and a viscous fluid that can dissipate energy. Moreover, such models are used in practice and for this we refer to [Fung 1993], where the author proposed such models to describe the response of biological matter which exhibits viscoelastic response, and to [Ramberg and Osgood 1943], where the authors deal with the inelastic response of bodies wherein a linearized measure of strain is related nonlinearly to stress.

Let us now formulate precise assumptions on $\mathbf{G}$ and $\mathbf{H}$. We assume that $\mathbf{H}, \mathbf{G}$ : $\mathbb{R}_{\text {sym }}^{2 \times 2} \rightarrow \mathbb{R}_{\text {sym }}^{2 \times 2}, \mathbf{H}, \mathbf{G} \in \mathscr{C}^{0,1}\left(\mathbb{R}_{\text {sym }}^{2 \times 2}\right)^{2 \times 2}, \mathbf{G}(\mathbf{0})=\mathbf{0}, \mathbf{H}(\mathbf{0})=\mathbf{0}$, and that there exists a function $F:[0,+\infty) \rightarrow[0,+\infty)$ such that the potential $\Phi(\mathbf{D}):=F(|\mathbf{D}|)$ for $\mathbf{D} \in \mathbb{R}_{\text {sym }}^{2 \times 2}$ satisfies $\mathbf{G}=\partial \Phi / \partial \mathbf{D}$. Moreover we assume the existence of $r \in[2, \infty)$ and positive constants $v_{0}, v_{1}$ and $v_{2}$ such that

$$
\begin{aligned}
v_{0}\left(1+|\mathbf{D}|^{2}\right)^{(r-2) / 2}|\mathbf{B}|^{2} & \leq \frac{\partial \mathbf{G}(\mathbf{D})}{\partial \mathbf{D}}: \mathbf{B} \otimes \mathbf{B} \leq v_{1}\left(1+|\mathbf{D}|^{2}\right)^{(r-2) / 2}|\mathbf{B}|^{2}, \\
\left|\frac{\partial \mathbf{H}(\mathbf{D})}{\partial \mathbf{D}}\right| & \leq v_{2}
\end{aligned}
$$

for all $\mathbf{B} \in \mathbb{R}_{\mathrm{sym}}^{2 \times 2}$ and almost all $\mathbf{D} \in \mathbb{R}_{\mathrm{sym}}^{2 \times 2}$. The prototypical example of the model we are interested in is given by

$$
\mathbf{G}(\mathbf{D})=\left(1+|\mathbf{D}|^{2}\right)^{(r-2) / 2} \mathbf{D}, \quad \mathbf{H}(\mathbf{D})=\left(1+|\mathbf{D}|^{2}\right)^{(q-2) / 2} \mathbf{D}
$$

with some $r \geq 2, q \in(1,2]$. For (1-8) it is easy to verify (1-6) and (1-7). Note that (1-7) allows one to consider more general examples than that introduced in (1-8). It is worth noticing that (1-7) says only that $\mathbf{H}$ is uniformly Lipschitz continuous but does not require any additional structure assumption as potentiality or monotonicity.

Concerning the boundary condition, we restrict ourselves to periodic (with respect to $\Omega$ ) boundary conditions that require some normalization condition (in order to guarantee uniqueness of a solution). For simplicity we choose the simplest one:

$$
\int_{\Omega} \rho_{0}(x) \boldsymbol{u}(t, x) d x=\int_{\Omega} \rho_{0}(x) \boldsymbol{u}_{t}(t, x) d x=\mathbf{0} \quad \text { for all } t \in(0, T) .
$$

A direct consequence of (1-9) is that we need to assume a compatibility condition on the data, namely, for all $t \in(0, T)$ we need that

$$
\int_{\Omega} \rho_{0}(x) \boldsymbol{f}(t, x) d x=\int_{\Omega} \rho_{0}(x) \boldsymbol{v}_{0}(x) d x=\int_{\Omega} \rho_{0}(x) \boldsymbol{u}_{0}(x) d x=\mathbf{0} .
$$

Although we use the simplest possible boundary condition we believe that our result can be adopted to a more general setting with more reasonable physical boundary data.

Next, we introduce the assumption put on the data of (1-1). For the density $\rho_{0}$, we assume that there are $0<\rho_{*} \leq \rho^{*}<\infty$ such that

$$
\rho_{0} \in L^{\infty}, \quad \rho_{*} \leq \rho_{0}(x) \leq \rho^{*} \quad \text { for almost all } x \in \Omega .
$$


Concerning the density of the external body forces we prescribe

$$
\rho_{0} f \in\left(L^{r}\left(0, T ;\left(W_{\text {per }}^{1, r}\right)^{2}\right)\right)^{*} .
$$

Finally, for the initial displacement and the initial velocity we assume that in addition to (1-10) they also satisfy

$$
\boldsymbol{u}_{0} \in\left(W_{\text {per }}^{1,2}\right)^{2}, \quad \boldsymbol{v}_{0} \in\left(L^{2}\right)^{2} .
$$

The existence of a weak solution for the problem (1-1) with nonlinear $\mathbf{T}_{v}$ satisfying (1-6) with $r=2$ can be found in [Friedman and Nečas 1988], [Demoulini 2000] and [Tvedt 2008] under certain structural assumptions on $\mathbf{T}$ that are more general than (1-3), (1-4) and (1-5). Next, the existence theory was extended for $r \geq 2$ in [Bulíček et al. 2012], where the authors assumed that the Cauchy stress satisfies (1-3)-(1-7). In addition they showed the uniqueness of a solution

$$
\boldsymbol{u} \in W^{1, \infty}\left(0, T ;\left(L^{2}\right)^{2}\right) \cap W^{1, r}\left(0, T ;\left(W^{1, r}\right)^{2}\right)
$$

to (1-1). Although all results in [Bulíček et al. 2012] treat the case of mixed boundary conditions, the method presented there works also in the easier periodic case in which we are interested in here. Moreover, assuming that the data are smooth, one can prove by the method introduced there that the unique solution $\boldsymbol{u}$ to (1-1) is more regular. We state the result in the next theorem.

Theorem 1.1 [Bulíček et al. 2012]. Let $r \geq 2, T>0$ be arbitrary. Assume that $\mathbf{T}$ satisfies (1-3)-(1-7) and the data ( $\left.\rho_{0}, \boldsymbol{f}, \boldsymbol{u}_{0}, \boldsymbol{v}_{0}\right)$ satisfy (1-10), (1-11)-(1-13). Then there exists a unique weak solution $\boldsymbol{u} \in W^{1, \infty}\left(0, T ;\left(L^{2}\right)^{2}\right) \cap W^{1, r}\left(0, T ;\left(W_{\text {per }}^{1, r}\right)^{2}\right)$ of (1-1).

In addition, assume that there is $p>2$ such that the data fulfill

$$
\begin{aligned}
\left(\rho_{0}, \boldsymbol{u}_{0}, \boldsymbol{v}_{0}\right) & \in W_{\text {per }}^{1, p} \times\left(W_{\text {per }}^{2, p}\right)^{2} \times\left(W_{\text {per }}^{2, p}\right)^{2}, \\
\boldsymbol{f} & \in W^{1,2}\left(0, T ;\left(L^{p}\right)^{2}\right) .
\end{aligned}
$$

Then the weak solution satisfies

$$
\begin{aligned}
\left(1+\left|\mathbf{D}\left(\boldsymbol{u}_{t}\right)\right|\right)^{(r-2) / 2} \mathbf{D}\left(\nabla \boldsymbol{u}_{t}\right) & \in L^{2}\left(0, T ;\left(L^{2}\right)^{2 \times 2 \times 2}\right), \\
\left(1+\left|\mathbf{D}\left(\boldsymbol{u}_{t}\right)\right|\right)^{(r-2) / 2} \mathbf{D}\left(\boldsymbol{u}_{t t}\right) & \in L^{2}\left(0, T ;\left(L^{2}\right)^{2 \times 2}\right), \\
\boldsymbol{u}_{t t} & \in L^{r^{\prime}}\left(0, T ;\left(L^{r^{\prime}}\right)^{2}\right) .
\end{aligned}
$$

The main result of our paper is that we improve (1-16) and get the Hölder continuity of the velocity gradient. Consequently, we use such information to obtain that the unique weak solution is in fact a classical one provided that the data are sufficiently smooth. The first improvement is this: 
Theorem 1.2. Let $r \geq 2, T>0$ be arbitrary. Assume that $\mathbf{T}$ satisfies (1-3)-(1-7) and the data $\left(\rho_{0}, \boldsymbol{f}, \boldsymbol{u}_{0}, \boldsymbol{v}_{0}\right)$ satisfy (1-10), (1-11)-(1-13). In addition, assume that there is $p>2$ such that (1-15) holds. Then there exists some $s \in(2, p)$ such that the unique solution of (1-1) satisfies

$$
\boldsymbol{u}_{t} \in W^{1, \infty}\left(0, T ;\left(L^{s}\right)^{2}\right) \cap L^{\infty}\left(0, T ;\left(W_{\mathrm{per}}^{2, s}\right)^{2}\right) .
$$

Consequently, for all $\alpha \in(0,(1-2 / s) / 3)$,

$$
\nabla \boldsymbol{u}_{t} \in\left(\mathscr{C}^{0, \alpha}(Q)\right)^{2 \times 2} .
$$

As a consequence of Theorem 1.2 we obtain:

Theorem 1.3. Let all assumptions of Theorem 1.2 hold. Then the unique solution from Theorem 1.2 satisfies

$$
\boldsymbol{u}_{t} \in W^{1, p}\left(0, T ;\left(L^{p}\right)^{2}\right) \cap L^{p}\left(0, T ;\left(W_{\text {per }}^{2, p}\right)^{2}\right) .
$$

If we in addition assume that

$$
\begin{gathered}
\left(\rho_{0}, \boldsymbol{u}_{0}, \boldsymbol{v}_{0}\right) \in W^{1, \infty} \times\left(W_{\text {per }}^{3, p}\right)^{2} \times\left(W_{\text {per }}^{3, p}\right)^{2}, \\
\boldsymbol{f} \in L^{p}\left(0, T ;\left(W_{\text {per }}^{1, p}\right)^{2}\right), \quad \mathbf{G}, \mathbf{H} \in \mathscr{C}_{\text {loc }}^{1,1}\left(\mathbb{R}_{\text {sym }}^{2 \times 2}\right)^{2 \times 2},
\end{gathered}
$$

then the unique solution from Theorem 1.2 satisfies

$$
\nabla \boldsymbol{u}_{t} \in W^{1, p}\left(0, T ;\left(L^{p}\right)^{2 \times 2}\right) \cap L^{p}\left(0, T ;\left(W_{\text {per }}^{2, p}\right)^{2 \times 2}\right) .
$$

As an immediate consequence of Theorem 1.3 and an interpolation Lemma A.1 we get:

Corollary 1.1. Let all assumptions of Theorem 1.3 hold with some $p>4$ and $\boldsymbol{f} \in \mathscr{C}(Q)$. Then the unique weak solution $\boldsymbol{u}$ is a classical one.

For general systems of partial differential equations Hölder continuity of weak solutions is a rare phenomenon, that can be obtained only under special circumstances. One of them is that if $\Omega \subset \mathbb{R}^{2}$ is as in Theorem 1.2. As far as we know the only former result in this direction for the problem (1-1) is the one from [Friedman and Nečas 1988] where Theorem 1.2 is proved in the case $r=2$. Another special condition when regularity (1-18) can be obtained is a special structure of the elliptic term $\mathbf{T}_{v}$. If it is assumed that the system (1-1) is a linear system of equations, i.e., classical Kelvin-Voigt model, one can establish the existence of a unique smooth solution (provided that data are smooth) by standard results for linear systems. In [DiBenedetto and Friedman 1984; 1985] a nonlinear function $\mathbf{G}$ is treated with the structure very similar to the one suggested in $(1-8)$ but the symmetric gradient is replaced with the full gradient, i.e., $\mathbf{T}_{v}=\mathbf{G}\left(\nabla \boldsymbol{u}_{t}\right)$. It is a remarkable fact that the method from [DiBenedetto and Friedman 1984; 1985] cannot be applied in the situation of (1-5), i.e., if the elliptic term depends only on $\mathbf{D}\left(\boldsymbol{u}_{t}\right)$. According to 
our best knowledge no results about the Hölder continuity of gradients of weak solutions are known if $\Omega \subset \mathbb{R}^{d}, d>2$ and the elliptic part of the equation depends only on the symmetric part of $\nabla \boldsymbol{u}_{t}$.

The method of the proof of Theorem 1.2 is based on the fact that a small improvement of regularity in (1-16) gives Hölder continuity of $\nabla \boldsymbol{u}_{t}$. This was first observed in [Boyarskiı̌ 1957] and [Meyers 1963] in the stationary case and extended to parabolic systems in [Nečas and Šverák 1991] and [Frehse and Seregin 1999]. This method was used in [Friedman and Nečas 1988] to prove Theorem 1.2 for $r=2$. First the integrability of $\boldsymbol{u}_{t t}$ was improved and then the system was treated as an elliptic one on time levels. This method must be modified if $r>2$ as in this case we do not know how to get separately only information about $\boldsymbol{u}_{t t}$. Regularity of $\boldsymbol{u}_{t t}$ and $\nabla^{2} \boldsymbol{u}_{t}$ must be dealt with simultaneously as it was suggested for generalized Navier-Stokes system in [Kaplický et al. 2002]. This is also the approach that we adopt here to prove Theorem 1.2.

The paper has the following structure. In the next section we introduce some auxiliary lemmas about linear stationary and parabolic systems with bounded measurable coefficients. The proofs can be found in the Appendix. In Section 3 we provide the proof of Theorem 1.2 if $r=2$. This result is not new, but it is a basis for the analysis in Section 4 where Theorem 1.2 is proved for $r>2$. Finally, we present a sketch of the proof of Theorem 1.3 in Section 5.

In the paper we use standard notation for Lebesgue and Sobolev spaces and their norms. If the domain on which the functions are considered is $\Omega=(0,1)^{2}$, we shorten the notation and write only $W^{1, q}, L^{q}$ or $\|\cdot\|_{q},\|\cdot\|_{1, q}$. The subscript per denotes periodicity with respect to $\Omega$. Particularly, $W_{\text {per }}^{1, q}$ are spaces of functions from $W_{\text {loc }}^{1, q}\left(\mathbb{R}^{2}\right)$ for which there is a representative that is periodic with respect to $\Omega$. Moreover, scalar-, vector- and tensor-valued functions are denoted by small letters, small bold letters and bold capital letters in what follows. Also in order to distinguish between scalars, vectors and tensors we use the abbreviations $X^{d}$ and $X^{d \times d}$ for vector- and tensor-valued function in a Banach space $X$. The symbol $\mathbb{R}_{\text {sym }}^{2 \times 2}$ denotes the space of all symmetric $2 \times 2$ matrices and for $\xi \in \mathbb{R}^{2 \times 2}, \boldsymbol{\xi}_{\text {sym }}$ is its symmetric part. For a function $\mathbf{G}: \mathbb{R}_{\text {sym }}^{2 \times 2} \rightarrow \mathbb{R}^{2 \times 2}$ we denote its gradient by $\partial_{\mathbf{D}} \mathbf{G}$. Then for any $\mathbf{B}, \mathbf{D} \in \mathbb{R}_{\mathrm{sym}}^{2 \times 2}$ we denote by $\partial_{\mathbf{D}} \mathbf{G}(\mathbf{D}): \mathbf{B} \otimes \mathbf{B}$ a scalar product of the matrices $\partial_{\mathbf{D}} \mathbf{G}(\mathbf{D})$ and $\mathbf{B} \otimes \mathbf{B}$. Symbols $\boldsymbol{u}_{t}$ and $\partial_{t} \boldsymbol{u}$ denote derivative of $\boldsymbol{u}$ with respect to $t \in(0, T)$.

\section{Auxiliary results}

In this section we recall some results for a linear system similar to (1-1), the proof of these results can be found in the Appendix. This linear system will play a crucial role in the proof of Theorem 1.2, where it will be used as the comparison problem. 
Lemma 2.1. Let $T>0$ be given and assume that $\mathbf{A}:(0, T) \times \Omega \rightarrow \mathbb{R}^{2 \times 2 \times 2 \times 2}$ is a measurable tensor-valued function satisfying for some $0<\lambda_{1} \leq \lambda_{2}<\infty$ and for almost all $(t, x) \in(0, T) \times \Omega$ the following symmetry and ellipticity conditions:

$$
\mathbf{A}_{i j}^{k l}(t, x)=\mathbf{A}_{k l}^{i j}(t, x)=\mathbf{A}_{k l}^{j i}(t, x) \quad \text { for all } i, j, k, l=1, \ldots, 2,
$$

$$
\lambda_{1}|\mathbf{D}|^{2} \leq \sum_{i, j, k, l=1}^{2} \mathbf{A}_{k l}^{i j}(t, x) \mathbf{D}_{i j} \mathbf{D}_{k l} \leq \lambda_{2}|\mathbf{D}|^{2} \quad \text { for all } \mathbf{D} \in \mathbb{R}_{\mathrm{sym}}^{2 \times 2}
$$

Then for any $\mathbf{F} \in L^{2}\left(0, T ;\left(L^{2}\right)^{2 \times 2}\right)$ and any $\Omega$-periodic $\boldsymbol{w}_{0} \in\left(L^{2}\right)^{2}$ having zero mean value, a unique $\Omega$-periodic weak solution

$$
\boldsymbol{w} \in \mathscr{C}\left(0, T ;\left(L^{2}\right)^{2}\right) \cap L^{2}\left(0, T ;\left(W^{1,2}\right)^{2}\right), \quad \int_{\Omega} \boldsymbol{w}(t, x) d x=\mathbf{0}
$$

exists to the problem

$$
\begin{aligned}
\boldsymbol{w}_{t}-\operatorname{div}(\mathbf{A D}(\boldsymbol{w})) & =-\operatorname{div} \mathbf{F} & & \text { in }(0, T) \times \Omega, \\
\boldsymbol{w}(0, \cdot) & =\boldsymbol{w}_{0}(\cdot) & & \text { in } \Omega .
\end{aligned}
$$

Moreover, there exist positive constants $K, L>0$ that are independent of $T, \mathbf{A}$ and $\mathbf{F}$ such that, for all s satisfying

$$
2 \leq s \leq 2+\frac{L \lambda_{1}}{\lambda_{2}}
$$

the following estimate holds:

$$
\sup _{t \in(0, T)}\|\boldsymbol{w}(t)\|_{s}^{2} \leq K\left(\frac{1}{\lambda_{1}}\|\mathbf{F}\|_{L^{2}\left(0, T ; L^{s}\right)}^{2}+\left\|\boldsymbol{w}_{0}\right\|_{s}^{2}\right) .
$$

In case one replaces $\mathbf{D}(\boldsymbol{w})$ by $\nabla \boldsymbol{w}$ in Lemma 2.1, the statement was proved in [Nečas and Šverák 1991]. However, following the procedure in that paper almost step by step one can prove Lemma 2.1 in full generality; see the Appendix for a detailed proof.

Note that in the previous lemma we did not improve the estimate for the gradient of the solution. As it is usual in parabolic equations the information on the spatial gradient of the solution will be deduced by comparing the equation with its steady form. Therefore, we recall the following lemma; see for example [Nečas 1967].

Lemma 2.2. Let $\mathbf{A}: \Omega \rightarrow \mathbb{R}^{2 \times 2 \times 2 \times 2}$ be a measurable tensor-valued function satisfying, for some $0<\lambda_{1} \leq \lambda_{2}<\infty$ and almost all $x \in \Omega$,

$$
\mathbf{A}_{i j}^{k l}(x)=\mathbf{A}_{k l}^{i j}(x)=\mathbf{A}_{k l}^{j i}(x) \quad \text { for all } i, j, k, l=1, \ldots, 2,
$$

$$
\lambda_{1}|\mathbf{D}|^{2} \leq \sum_{i, j, k, l=1}^{2} \mathbf{A}_{k l}^{i j}(x) \mathbf{D}_{i j} \mathbf{D}_{k l} \leq \lambda_{2}|\mathbf{D}|^{2} \quad \text { for all } \mathbf{D} \in \mathbb{R}_{\mathrm{sym}}^{2 \times 2} .
$$


Then, for any $\mathbf{F} \in\left(L^{2}\right)^{2 \times 2}$, there exists a unique $\Omega$-periodic weak solution $\boldsymbol{w} \in$ $\left(W^{1,2}\right)^{2}$ such that $\int_{\Omega} \boldsymbol{w} d x=\mathbf{0}$ solving the problem

$$
-\operatorname{div}(\mathbf{A D}(\boldsymbol{w}))=-\operatorname{div} \mathbf{F} \quad \text { in } \Omega .
$$

Moreover, there exist $K, L>0$ independent of $T, \mathbf{A}$ and $\mathbf{F}$ such that, for all

$$
2 \leq s \leq 2+\frac{L \lambda_{1}}{\lambda_{2}}
$$

we have

$$
\|\mathbf{D}(\boldsymbol{w})\|_{s} \leq \frac{K}{\lambda_{1}}\|\mathbf{F}\|_{s} .
$$

In general the constants $K, L$ from Lemma 2.1 and Lemma 2.2 may be different but without loss of generality we assume in what follows that they are the same.

\section{Proof of Theorem 1.2 in the case $r=2$}

This section is devoted to the proof of Theorem 1.2 for $r=2$. First, we introduce an $\varepsilon$-approximation to the problem (1-1), but we still write $\boldsymbol{u}$ instead of $\boldsymbol{u}^{\varepsilon}$ for its solution:

$$
\rho_{0} \boldsymbol{u}_{t t}-\operatorname{div}\left(\mathbf{G}\left(\mathbf{D}\left(\boldsymbol{u}_{t}\right)\right)\right)=\rho_{0} \boldsymbol{f}+\operatorname{div}\left(\mathbf{H}\left(\mathbf{D}\left(\boldsymbol{u} \star \omega^{\varepsilon}\right)\right) \quad \text { in }(0, T) \times \Omega,\right.
$$

with periodic boundary condition and initial data $\left(\boldsymbol{u}_{0}, \boldsymbol{v}_{0}\right)$. Here $\omega: \mathbb{R}^{2} \rightarrow \mathbb{R}$ is a standard regularizing kernel, i.e., $\omega \in C_{0}^{\infty}(U(0,1))$ is nonnegative, radially symmetric, $\int_{\mathbb{R}^{2}} \omega d x=1$, and we define

$$
\omega^{\varepsilon}(x)=\varepsilon^{-2} \omega\left(\frac{x}{\varepsilon}\right) .
$$

Note that the convolution in the last term of (3-1) is taken only in space direction. Next, we formulate the existence result for (3-1), that is the starting point of our analysis.

Lemma 3.1. Let $\mathbf{H}$ and $\mathbf{G}$ satisfy (1-6)-(1-7) with $r=2$. Assume that $\rho_{0}, \boldsymbol{f}, \boldsymbol{u}_{0}$ and $\boldsymbol{v}_{0}$ satisfy (1-10) and (1-11)-(1-13). In addition, assume that $\boldsymbol{f} \in W^{1,2}\left(0, T ;\left(L^{2}\right)^{2}\right)$, $\rho_{0} \in W_{\mathrm{per}}^{1,2+\delta}$ for a certain $\delta>0$ and $\boldsymbol{u}_{0}, \boldsymbol{v}_{0} \in\left(W_{\mathrm{per}}^{2,2}\right)^{2}$. Then for any $\varepsilon>0$ there exists a unique $\Omega$-periodic weak solution $\boldsymbol{u}$ to (3-1), (1-1) $2_{2}-(1-1)_{3}$ that obeys the a priori estimate

$$
\begin{aligned}
\left\|\nabla^{2} \boldsymbol{u}\right\|_{L^{\infty}\left(0, T ; L^{2}\right)}+\left\|\nabla \boldsymbol{u}_{t}\right\|_{L^{\infty}\left(I, L^{2}\right)}+\| \nabla & \boldsymbol{u}_{t}\left\|_{L^{2}\left(I, W^{1,2}\right)}+\right\| \boldsymbol{u}_{t t}\left\|_{L^{\infty}\left(I, L^{2}\right)}+\right\| \boldsymbol{u}_{t t} \|_{L^{2}\left(I, W^{1,2}\right)} \\
& \leq C_{1}\left(1+\left\|\mathbf{G}\left(\mathbf{D}\left(\boldsymbol{v}_{0}\right)\right)\right\|_{1,2}+\left\|\mathbf{H}\left(\mathbf{D}\left(\boldsymbol{u}_{0}\right)\right)\right\|_{1,2}\right),
\end{aligned}
$$

where $C_{1}>0$ is independent of $v_{1}$ and $\epsilon$. Moreover, this solution converges to the unique solution of (1-1) as $\varepsilon \rightarrow 0_{+}$. 
Proof. The proof is presented in [Bulíček et al. 2012] for the system (1-1) with mixed (Dirichlet and Neumann) boundary conditions. In our situation, smoothing of the term with $\mathbf{H}$ in (3-1) simplifies the situation and also the periodic boundary conditions are simpler to deal with. Since the proof of Lemma 3.1 follows [Bulíček et al. 2012, Theorem 4.1, p. 9] line by line we do not present it here.

Lemma 3.2. Let all the assumptions of Lemma 3.1 hold. Let $\varepsilon>0$ be arbitrary and $\boldsymbol{u}$ the unique weak solution to (3-1). Assume that for some $\delta>0$ and s satisfying

$$
2 \leq s \leq 2+\min \left(\frac{L v_{0} \rho_{*}}{v_{1} \rho^{*}}, \frac{\delta}{2}\right),
$$

the data fulfill

$$
\begin{aligned}
\left(\boldsymbol{u}_{0}, \boldsymbol{v}_{0}, \rho_{0}\right) & \in\left(W_{\text {per }}^{2, s}\right)^{2} \times\left(W_{\text {per }}^{2, s}\right)^{2} \times W_{\text {per }}^{1,2+\delta}(\Omega), \\
\boldsymbol{f} & \in W^{1,2}\left(0, T ;\left(L^{s}\right)^{2}\right) .
\end{aligned}
$$

Then the following estimate holds:

$$
\sup _{t \in(0, T)}\left\|\boldsymbol{u}_{t t}\right\|_{s} \leq\left(1+v_{1}\right) C\left(\boldsymbol{u}_{0}, \boldsymbol{v}_{0}, \delta, \boldsymbol{f}, v_{0}, v_{2}\right) .
$$

Proof. First, we construct an $\Omega$-periodic F having zero mean value over $\Omega$ such that

$$
\operatorname{div} \mathbf{F}=\rho_{0} f \quad \text { in }(0, T) \times \Omega .
$$

Such a construction is possible due to the compatibility condition (1-10). Moreover, using the theory for the divergence equation (see for example [Feireisl and Novotný 2009; Novotný and Straškraba 2004]) and (1-11) we have

$$
\|\mathbf{F}\|_{W^{1,2}\left(0, T ; W^{1, s}\right)} \leq C\left\|\rho_{0} f\right\|_{W^{1,2}\left(0, T ; L^{s}\right)} \leq C,
$$

where the last inequality follows from (3-4). Next, we set $\boldsymbol{w}:=\rho_{0} \boldsymbol{u}_{t t}$ and applying $\partial_{t}$ to (3-1) (in view of (3-2), this procedure is rigorous) we see that $\boldsymbol{w}$ is a weak solution of the system

$$
\boldsymbol{w}_{t}-\operatorname{div}(\mathbf{A D}(\boldsymbol{w}))=\operatorname{div} \tilde{\mathbf{F}} \quad \text { in }(0, T) \times \Omega,
$$

where

$$
\begin{aligned}
\mathbf{A} & :=\frac{1}{\rho_{0}} \frac{\partial \mathbf{G}\left(\mathbf{D}\left(\boldsymbol{u}_{t}\right)\right)}{\partial \mathbf{D}}, \\
\tilde{\mathbf{F}} & :=\mathbf{F}_{t}+\frac{\partial \mathbf{H}\left(\mathbf{D}\left(\boldsymbol{u} \star \omega^{\varepsilon}\right)\right)}{\partial \mathbf{D}} \mathbf{D}\left(\boldsymbol{u}_{t} \star \omega^{\varepsilon}\right)-\left[\frac{\partial \mathbf{G}\left(\mathbf{D}\left(\boldsymbol{u}_{t}\right)\right)}{\partial \mathbf{D}}\right]\left(\frac{\nabla \rho_{0}}{\rho_{0}} \otimes \boldsymbol{u}_{t t}\right) .
\end{aligned}
$$

Since $\mathbf{G}$ is assumed to satisfy (1-6) with $r=2$, and $\rho_{0}$ satisfies (1-11), we see that the matrix $\mathbf{A}$ fulfills (2-1)-(2-2) with

$$
\lambda_{1}:=\frac{\nu_{0}}{\rho^{*}} \quad \text { and } \quad \lambda_{2}:=\frac{\nu_{1}}{\rho_{*}} .
$$


Hence, assuming that $s$ satisfies (3-3), it also satisfies $s \in\left[2,2+L \lambda_{1} / \lambda_{2}\right]$ and we can use Lemma 2.1 to deduce that

$$
\sup _{t \in(0, T)}\|\boldsymbol{w}(t)\|_{s}^{2} \leq K\left(\|\boldsymbol{w}(0)\|_{s}^{2}+\frac{1}{\lambda_{1}} \int_{0}^{T}\|\tilde{\mathbf{F}}\|_{s}^{2}\right) .
$$

We check that the right side is finite. To see this, we first evaluate the initial value $\boldsymbol{w}(0)$. Using (3-1) we see that

$$
\boldsymbol{w}(0):=\rho_{0} \boldsymbol{u}_{t t}(0)=\operatorname{div}\left(\mathbf{G}\left(\mathbf{D}\left(\boldsymbol{v}_{0}\right)\right)+\mathbf{H}\left(\mathbf{D}\left(\boldsymbol{u}_{0} \star \omega^{\varepsilon}\right)\right)\right)+\rho_{0} \boldsymbol{f}(0)
$$

and by using (1-6)-(1-7) and (3-4) we obtain that (for estimating $f$ we use the embedding $W^{1,2}(0, T) \hookrightarrow \mathscr{C}^{0,1 / 2}([0, T]) \hookrightarrow \mathscr{C}([0, T])$ in dimension one $)$

$$
\begin{aligned}
\|\boldsymbol{w}(0)\|_{s} & \leq\left\|\operatorname{div} \mathbf{G}\left(\mathbf{D}\left(\boldsymbol{v}_{0}\right)\right)\right\|_{s}+\left\|\operatorname{div} \mathbf{H}\left(\mathbf{D}\left(\boldsymbol{u}_{0} \star \omega^{\varepsilon}\right)\right)\right\|_{s}+\left\|\rho_{0} \boldsymbol{f}(0)\right\|_{s} \\
& \leq v_{1}\left\|\boldsymbol{v}_{0}\right\|_{2, s}+v_{2}\left\|\boldsymbol{u}_{0}\right\|_{2, s}+\rho^{*}\|\boldsymbol{f}(0)\|_{s} \leq C\left(1+v_{1}\right) .
\end{aligned}
$$

It remains to estimate the norm of $\tilde{\mathbf{F}}$ appearing on the right side of (3-9). Using (3-6) and (1-6)-(1-7) we obtain that

$$
\begin{aligned}
\int_{0}^{T}\|\tilde{\mathbf{F}}\|_{s}^{2} & \leq \int_{0}^{T}\left(\left\|\mathbf{F}_{t}\right\|_{s}^{2}+v_{2}\left\|\mathbf{D}\left(\boldsymbol{u}_{t}\right)\right\|_{s}^{2}+\left\|\rho_{0}^{-1} \partial_{\mathbf{D}} \mathbf{G}\left(\mathbf{D}\left(\boldsymbol{u}_{t}\right)\right) \nabla \rho_{0} \otimes \boldsymbol{u}_{t t}\right\|_{s}^{2}\right) \\
& \leq \int_{0}^{T}\left(\left\|\mathbf{F}_{t}\right\|_{1, s}^{2}+v_{2}\left\|\mathbf{D}\left(\boldsymbol{u}_{t}\right)\right\|_{s}^{2}+\frac{v_{1}^{2}}{\rho_{*}^{2}}\left\|\nabla \rho_{0}\right\|_{2+\delta}^{2}\left\|\boldsymbol{u}_{t t}\right\|_{s(2+\delta) /(2+\delta-s)}^{2}\right) \\
& \leq C\left(\boldsymbol{v}_{0}, \boldsymbol{u}_{0}, \boldsymbol{f}, \rho_{0}, \nu_{2}\right)+C\left(\rho_{0}, \delta\right) v_{1}^{2} \int_{0}^{T}\left\|\boldsymbol{u}_{t t}\right\|_{1,2}^{2} .
\end{aligned}
$$

Consequently, using the uniform estimate (3-2) we can bound the last term on the right side of (3-11) and inserting this and (3-10) into (3-9) we deduce (3-5).

Since, we already know that $\boldsymbol{u}_{t t}$ belongs to a better space than $L^{2}$ uniformly in time, we can improve the spatial regularity of $\boldsymbol{u}$ with help of Lemma 2.2.

Lemma 3.3. Let all assumptions of Lemma 3.1 hold. Then for any $\varepsilon>0, \delta>0$ and $s>0$ fulfilling (3-3) and any data satisfying (3-4), the unique solution $\boldsymbol{u}$ to the problem (3-1) satisfies for almost all $t \in(0, T)$ the estimate: ${ }^{1}$

$$
\left\|\nabla^{2} \boldsymbol{u}_{t}(t)\right\|_{s} \leq C\left(1+\left\|\boldsymbol{u}_{t t}\right\|_{L^{\infty}\left(0, T ; L^{s}\right)}+\left\|\nabla^{2}\left(\boldsymbol{u}(t) \star \omega^{\varepsilon}\right)\right\|_{s}\right),
$$

with $C$ depending only on $\left(\rho_{0}, \boldsymbol{f}, \boldsymbol{v}_{0}, \boldsymbol{u}_{0}, v_{0}, v_{2}, T\right)$.

Proof. Since we know from Lemma 3.1 that (3-1) holds pointwise at almost all time levels $t \in(0, T)$. We fix such an arbitrary $t \in(0, T)$ and rewrite the problem

\footnotetext{
${ }^{1}$ The right side of (3-12) is finite, since for the time derivative we have an estimate due to Lemma 3.2 and the last term in (3-12) is finite due to regularization.
} 
(3-1) as

$$
-\operatorname{div}\left(\mathbf{G}\left(\mathbf{D}\left(\boldsymbol{u}_{t}\right)\right)\right)=\operatorname{div}\left(\mathbf{F}+\mathbf{H}\left(\mathbf{D}\left(\boldsymbol{u} \star \omega^{\varepsilon}\right)\right)-\mathbf{F}_{0}\right) \quad \text { in } \Omega,
$$

where $\mathbf{F}_{0}$ is found such that (note that $t$ is fixed in what follows)

$$
\operatorname{div} \mathbf{F}_{0}=\rho_{0} \boldsymbol{u}_{t t} \quad \text { in } \Omega, \quad\left\|\mathbf{F}_{0}(t)\right\|_{1, s} \leq C \rho^{*}\left\|\boldsymbol{u}_{t t}(t)\right\|_{s}
$$

and $\mathbf{F}$ satisfies

$$
\operatorname{div} \mathbf{F}=\rho_{0} \boldsymbol{f} \quad \text { in } \Omega, \quad\|\mathbf{F}(t)\|_{1, s} \leq C \rho^{*}\|\boldsymbol{f}(t)\|_{s} .
$$

Next, we fix $k \in\{1,2\}$, denote $\boldsymbol{w}:=\partial_{k} \boldsymbol{u}_{t}$ and

$$
\mathbf{A}:=\frac{\partial \mathbf{G}\left(\mathbf{D}\left(\boldsymbol{u}_{t}\right)\right)}{\partial \mathbf{D}}
$$

and differentiate (3-13) in the weak sense with respect to $x_{k}$. We obtain the system of equations

$$
-\operatorname{div}(\mathbf{A D}(\boldsymbol{w}))=\operatorname{div}\left(\partial_{k} \mathbf{F}+\partial_{k} \mathbf{H}\left(\mathbf{D}\left(\boldsymbol{u} \star \omega^{\varepsilon}\right)\right)-\partial_{k} \mathbf{F}_{0}\right) \quad \text { in } \Omega,
$$

equipped with periodic boundary conditions and requiring zero mean value for $\boldsymbol{w}$. Similarly as in the proof of Lemma 3.2, A satisfies the assumption of Lemma 2.2 with $\lambda_{1}:=v_{0}$ and $\lambda_{2}:=v_{1}$. Hence for any $s^{*} \in\left[2,2+L v_{0} / v_{1}\right]$ we have the estimate

$$
\|\mathbf{D}(\boldsymbol{w})\|_{s^{*}} \leq \frac{K}{v_{0}}\left(\|\mathbf{F}\|_{1, s^{*}}+\left\|\mathbf{F}_{0}\right\|_{1, s^{*}}+\left\|\mathbf{H}\left(\mathbf{D}\left(\boldsymbol{u} \star \omega^{\varepsilon}\right)\right)\right\|_{1, s^{*}}\right) .
$$

Since, we know that $s \leq 2+L\left(v_{0} \rho_{*}\right) /\left(v_{1} \rho^{*}\right) \leq 2+L v_{0} / v_{1}$, we see that (3-17) also holds for $s^{*}:=s$. Moreover, since it holds for any $k=1,2$ we can deduce from (3-17) by using the definition of $\mathbf{F}$ and $\boldsymbol{F}_{0}$ that

$$
\left\|\nabla^{2} \boldsymbol{u}_{t}(t)\right\|_{s} \leq \frac{C\left(\rho_{0}\right)}{v_{0}}\left(\|\boldsymbol{f}(t)\|_{s}+\left\|\boldsymbol{u}_{t t}(t)\right\|_{s}+v_{2}\left\|\mathbf{D}\left(\boldsymbol{u} \star \omega^{\varepsilon}(t)\right)\right\|_{1, s}\right) .
$$

Consequently, using (3-4), (3-3) and the a priori uniform estimates (3-2), we deduce (3-12).

Having all previous estimates, we are ready to prove Theorem 1.2 for $r=2$. Since, the case $r=2$ will be used in the proof of Theorem 1.2 for $r>2$ we formulate it as a special theorem where we trace the important constant $v_{1}$.

Theorem 3.1. Let $T>0$ be arbitrary. Assume that $\mathbf{T}$ satisfies (1-3)-(1-7) with $r=2$ and that data $\left(\rho_{0}, \boldsymbol{f}, \boldsymbol{u}_{0}, \boldsymbol{v}_{0}\right)$ satisfy (1-10), (1-11)-(1-13). In addition, assume that there is $p>2$ such that the data fulfill

$$
\begin{aligned}
\left(\boldsymbol{u}_{0}, \boldsymbol{v}_{0}, \rho_{0}\right) & \in\left(W_{\text {per }}^{2, p}\right)^{2} \times\left(W_{\text {per }}^{2, p}\right)^{2} \times W_{\text {per }}^{1, p}(\Omega), \\
\boldsymbol{f} & \in W^{1,2}\left(0, T ;\left(L^{p}\right)^{2}\right) .
\end{aligned}
$$


Then there exists a constant $C$ depending only on $\left(\rho_{0}, \boldsymbol{v}_{0}, \boldsymbol{u}_{0}, \boldsymbol{f}, T, v_{0}, v_{2}, p\right)$ such that for any s fulfilling

$$
2 \leq s \leq 2+\min \left(\frac{L v_{0} \rho_{*}}{v_{1} \rho^{*}}, \frac{p-2}{2}\right),
$$

the unique weak solution (1-1) satisfies the estimate

$$
\left\|\boldsymbol{u}_{t t}\right\|_{L^{\infty}\left(0, T ; L^{s}\right)}+\left\|\nabla^{2} \boldsymbol{u}_{t}\right\|_{L^{\infty}\left(0, T ; L^{s}\right)} \leq C\left(1+v_{1}\right) .
$$

Proof. To prove the theorem it is enough to show estimate (3-21) for the unique solutions of the approximating problem (3-1). Indeed, having uniform ( $\varepsilon$-independent) estimate (3-21) for the solution of the approximate problem it is easy to let $\epsilon \rightarrow 0+$ and to obtain a solution of the original problem (1-1). The estimate (3-21) is valid for this solution due to the weak*-lower semicontinuity of the norm in $L^{\infty}\left(0, T ; L^{s}\right)$. Uniqueness of the solution follows by the method of [Bulíček et al. 2012]; compare Lemma 3.1. Due to our assumption on the data and $s$ we see that also all assumptions of Lemmas 3.1-3.3 are satisfied. We can use (3-12) to prove (3-21). To do so, we need to estimate the last two terms on the right side of (3-12). Note that both of them are finite, so we directly have an estimate of the form (3-21) but with right side depending on $\varepsilon$. To avoid this dependence we estimate both terms as follows. We start with the time derivative for which we obtain by direct use of Lemma 3.2 that

$$
\left\|\boldsymbol{u}_{t t}\right\|_{L^{\infty}\left(0, T ; L^{s}\right)} \leq C\left(1+v_{1}\right) .
$$

Next, for the second term, we get, by (3-19),

$$
\begin{aligned}
\left\|\nabla^{2}\left(\boldsymbol{u}(t) \star \omega^{\varepsilon}\right)\right\|_{s} & \leq C\left\|\nabla^{2} \boldsymbol{u}(t)\right\|_{s}=C\left\|\int_{0}^{t} \nabla^{2} \boldsymbol{u}_{t}(\tau) d \tau+\nabla^{2} \boldsymbol{u}_{0}\right\|_{s} \\
& \leq C\left(1+\int_{0}^{t}\left\|\nabla^{2} \boldsymbol{u}_{t}(\tau)\right\|_{s} d \tau\right) .
\end{aligned}
$$

Using (3-22) and (3-23), we see that (3-12) reduces to

$$
\left\|\nabla^{2} \boldsymbol{u}_{t}(t)\right\|_{s} \leq C\left(1+v_{1}+\int_{0}^{t}\left\|\nabla^{2} \boldsymbol{u}_{t}(\tau)\right\|_{s} d \tau\right) .
$$

Applying Gronwall's lemma in its integral form, we deduce (3-21).

\section{Proof of Theorem 1.2 in the case $r>2$}

This section is devoted to the proof of Theorem 1.2 for $r>2$. It is based on a direct application of the result from the previous section onto a suitable approximating problem. 
First, we introduce a quadratic approximation of the problem (1-1). For any $\lambda>1$ we define Lipschitz continuous functions $\eta_{\lambda}$ and $\mu_{\lambda}$ as follows:

$$
\begin{aligned}
& \eta_{\lambda}(s):= \begin{cases}1 & \text { for } s \in\left[0,2 \lambda^{2}\right], \\
-\frac{s-3 \lambda^{2}}{\lambda^{2}} & \text { for } s \in\left(2 \lambda^{2}, 3 \lambda^{2}\right), \\
0 & \text { for } s \geq 3 \lambda^{2},\end{cases} \\
& \mu_{\lambda}(s):= \begin{cases}0 & \text { for } s \in\left[0, \lambda^{2}\right], \\
\gamma_{\lambda} \frac{s-\lambda^{2}}{\lambda^{2}} & \text { for } s \in\left(\lambda^{2}, 2 \lambda^{2}\right), \\
\gamma_{\lambda} & \text { for } s \geq 2 \lambda^{2},\end{cases}
\end{aligned}
$$

with some constant $\gamma_{\lambda} \in \mathbb{R}_{+}$to be specified later. We approximate $\mathbf{G}$ by $\mathbf{G}^{\lambda}$ as

$$
\mathbf{G}^{\lambda}(\mathbf{D}):=\eta_{\lambda}\left(|\mathbf{D}|^{2}\right) \mathbf{G}(\mathbf{D})+\mu_{\lambda}\left(|\mathbf{D}|^{2}\right) \mathbf{D} .
$$

Note that for $\mathbf{G}^{\lambda}$ a potential can be constructed. The most important properties of this approximation are introduced in the following lemma.

Lemma 4.1. Let $\mathbf{G}$ satisfy the assumption (1-6) with $r>2$ and $\nu_{0}, v_{1}>0$. Let $\lambda>1$ be arbitrary. We set in (4-1) and (4-2)

$$
\gamma_{\lambda}:=7 \nu_{1}\left(1+3 \lambda^{2}\right)^{(r-2) / 2} .
$$

Then for all $\mathbf{B} \in \mathbb{R}_{\mathrm{sym}}^{2 \times 2}$ and almost all $\mathbf{D} \in \mathbb{R}_{\mathrm{sym}}^{2 \times 2}$ it holds

$$
\bar{v}_{0}|\mathbf{B}|^{2} \leq \partial_{\mathbf{D}} \mathbf{G}^{\lambda}(\mathbf{D}): \mathbf{B} \otimes \mathbf{B} \leq \bar{v}_{1}|\mathbf{B}|^{2}
$$

with $\bar{\nu}_{0}$ and $\bar{\nu}_{1}$ given as

$$
\begin{aligned}
& \bar{v}_{0}:=v_{0}, \\
& \bar{v}_{1}:=\bar{v}_{1}(\lambda):=36 v_{1}\left(1+3 \lambda^{2}\right)^{(r-2) / 2} .
\end{aligned}
$$

Moreover, setting $\bar{\lambda}(\mathbf{D}):=\min (\lambda,|\mathbf{D}|)$, we get

(4-8) $v_{0}\left(1+\bar{\lambda}(\mathbf{D})^{2}\right)^{(r-2) / 2}|\mathbf{B}|^{2} \leq \partial_{\mathbf{D}} \mathbf{G}^{\lambda}(\mathbf{D}): \mathbf{B} \otimes \mathbf{B} \leq 36 v_{1}\left(1+3 \bar{\lambda}(\mathbf{D})^{2}\right)^{(r-2) / 2}|\mathbf{B}|^{2}$.

Proof. To shorten the notation we write $\partial_{\mathbf{D}} \mathbf{G}^{\lambda}(\mathbf{D}): \mathbf{B} \otimes \mathbf{B}$ only as $\partial_{\mathbf{D}} \mathbf{G}(\mathbf{D}): \mathbf{B} \otimes \mathbf{B}$. Using the definition of $\mathbf{G}^{\lambda}$ we get

$$
\begin{aligned}
I=\eta_{\lambda}\left(|\mathbf{D}|^{2}\right) \partial_{\mathbf{D}} \mathbf{G}(\mathbf{D}): \mathbf{B} \otimes \mathbf{B}+2 \eta_{\lambda}^{\prime}\left(|\mathbf{D}|^{2}\right)(\mathbf{D} \cdot \mathbf{B})(\mathbf{G}(\mathbf{D}) \cdot \mathbf{B}) \\
+\mu_{\lambda}\left(|\mathbf{D}|^{2}\right)|\mathbf{B}|^{2}+2 \mu_{\lambda}^{\prime}\left(|\mathbf{D}|^{2}\right)(\mathbf{D} \cdot \mathbf{B})^{2} .
\end{aligned}
$$


From this identity and the definition of $\eta_{\lambda}$ and $\mu_{\lambda}$ we finally conclude that

$$
I= \begin{cases}\partial_{\mathbf{D}} \mathbf{G}(\mathbf{D}): \mathbf{B} \otimes \mathbf{B} & \text { if }|\mathbf{D}|^{2}<\lambda^{2}, \\ \partial_{\mathbf{D}} \mathbf{G}(\mathbf{D}): \mathbf{B} \otimes \mathbf{B}+\gamma_{\lambda} \frac{|\mathbf{D}|^{2}-\lambda^{2}}{\lambda^{2}}|\mathbf{B}|^{2}+\frac{2 \gamma_{\lambda}}{\lambda^{2}}(\mathbf{D} \cdot \mathbf{B})^{2} & \text { if }|\mathbf{D}|^{2} \in\left(\lambda^{2}, 2 \lambda^{2}\right), \\ -\frac{|\mathbf{D}|^{2}-3 \lambda^{2}}{\lambda^{2}} \partial_{\mathbf{D}} \mathbf{G}(\mathbf{D}): \mathbf{B} \otimes \mathbf{B}-\frac{2}{\lambda^{2}}(\mathbf{D} \cdot \mathbf{B})(\mathbf{G}(\mathbf{D}) \cdot \mathbf{B})+\gamma_{\lambda}|\mathbf{B}|^{2} & \text { if }|\mathbf{D}|^{2} \in\left(2 \lambda^{2}, 3 \lambda^{2}\right), \\ \gamma_{\lambda}|\mathbf{B}|^{2} & \text { if }|\mathbf{D}|^{2}>3 \lambda^{2} .\end{cases}
$$

Now we remark that by the assumption (1-6) on $\mathbf{G}$ we get

$$
(\mathbf{G}(\mathbf{D}) \cdot \mathbf{B}) \leq v_{1}\left(1+|\mathbf{D}|^{2}\right)^{(r-2) / 2}|\mathbf{B}||\mathbf{D}| .
$$

Defining $Y:=I /|\mathbf{B}|^{2}$ and noting that $\lambda>1$, it follows that

$$
\begin{aligned}
v_{0}\left(1+|\mathbf{D}|^{2}\right)^{(r-2) / 2} & \leq Y \leq v_{1}\left(1+|\mathbf{D}|^{2}\right)^{(r-2) / 2} & & \text { if }|\mathbf{D}|^{2}<\lambda^{2}, \\
v_{0}\left(1+|\mathbf{D}|^{2}\right)^{(r-2) / 2} & \leq Y \leq v_{1}\left(1+|\mathbf{D}|^{2}\right)^{(r-2) / 2}+5 \gamma_{\lambda} & & \text { if }|\mathbf{D}|^{2} \in\left(\lambda^{2}, 2 \lambda^{2}\right), \\
\gamma_{\lambda}-6 v_{1}\left(1+|\mathbf{D}|^{2}\right)^{(r-2) / 2} & \leq Y \leq \gamma_{\lambda}+7 v_{1}\left(1+|\mathbf{D}|^{2}\right)^{(r-2) / 2} & & \text { if }|\mathbf{D}|^{2} \in\left(2 \lambda^{2}, 3 \lambda^{2}\right), \\
\gamma_{\lambda} & =Y & & \text { if }|\mathbf{D}|^{2}>3 \lambda^{2},
\end{aligned}
$$

and we see that (4-5)-(4-8) follows.

Next, we find $\lambda_{0}>1$ such that ${ }^{2} \min \left(L \bar{v}_{0} \rho_{*} /\left(\bar{v}_{1} \rho^{*}\right),(p-2) / 2\right)=L \bar{v}_{0} \rho_{*} /\left(\bar{v}_{1} \rho^{*}\right)$ and $\bar{\nu}_{1} \geq 1$ for all $\lambda>\lambda_{0}$.

Finally, for arbitrary fixed $\lambda>\lambda_{0}$, we consider an approximation of (1-1) of the form

$$
\begin{aligned}
\rho_{0} \boldsymbol{u}_{t t}-\operatorname{div} \mathbf{G}^{\lambda}\left(\mathbf{D}\left(\boldsymbol{u}_{t}\right)-\operatorname{div} \mathbf{H}(\mathbf{D}(\boldsymbol{u}))\right. & =\rho_{0} \boldsymbol{f} & & \text { in } Q, \\
\boldsymbol{u}(0, \cdot) & =\boldsymbol{u}_{0}(\cdot) & & \text { in } \Omega, \\
\boldsymbol{u}_{t}(0, \cdot) & =\boldsymbol{v}_{0}(\cdot) & & \text { in } \Omega, \\
\int_{\Omega} \rho_{0}(x) \boldsymbol{u}(t, x) d x=\int_{\Omega} \rho_{0}(x) \boldsymbol{u}_{t}(t, x) d x & =\mathbf{0} & & \text { for all } t \in(0, T),
\end{aligned}
$$

equipped with periodic boundary conditions for $\boldsymbol{u}$.

According to Lemma 4.1, $\mathbf{G}^{\lambda}$ satisfies all assumptions of Theorem 3.1 and we get that for all $s$ satisfying

$$
2 \leq s \leq 2+\frac{L \bar{v}_{0} \rho_{*}}{\bar{v}_{1} \rho^{*}}
$$

\footnotetext{
${ }^{2}$ The constant $p>2$ appears in Theorem 1.2 and it is assumed to be the same as in Theorem 3.1.
} 
the unique weak solution (4-9) satisfies the estimate

$$
\begin{aligned}
\left\|\nabla^{2} \boldsymbol{u}_{t}\right\|_{L^{\infty}\left(0, T ; L^{s}\right)} & \leq C\left(\rho_{0}, \boldsymbol{f}, T, \bar{v}_{0}, \nu_{2}, \boldsymbol{v}_{0}, \boldsymbol{u}_{0}, p\right)\left(\bar{v}_{1}+1\right) \\
& \leq C\left(\rho_{0}, \boldsymbol{f}, T, \bar{v}_{0}, \nu_{2}, \boldsymbol{v}_{0}, \boldsymbol{u}_{0}, p\right) \bar{v}_{1} .
\end{aligned}
$$

Using the definition of $\bar{v}_{0}$ and $\bar{\nu}_{1}$ we can set

$$
2 \leq s=2+R \lambda^{2-r}
$$

for a fixed $R \in\left(0, \frac{L \rho_{*} v_{0}}{36 \rho^{*} v_{1}}\left(\frac{1}{2}\right)^{r-2}\right)$ and rewrite the estimate (4-11) as

$$
\left\|\nabla^{2} \boldsymbol{u}_{t}\right\|_{L^{\infty}\left(0, T ; L^{s}\right)} \leq C\left(\rho_{0}, \boldsymbol{f}, T, \nu_{0}, \nu_{2}, \boldsymbol{v}_{0}, \boldsymbol{u}_{0}, p, r\right) \lambda^{r-2} .
$$

Our main goal, based on the estimate (4-13), is to find a sufficiently large $\lambda>\lambda_{0}$ such that

$$
M:=\left\|1+\bar{\lambda}\left(\mathbf{D}\left(\boldsymbol{u}_{t}\right)\right)^{2}\right\|_{L^{\infty}\left(0, T ; L^{\infty}\right)} \leq \lambda^{2} .
$$

For such $\lambda$ the equality $\mathbf{G}^{\lambda}\left(\mathbf{D}\left(\boldsymbol{u}_{t}\right)\right)=\mathbf{G}\left(\mathbf{D}\left(\boldsymbol{u}_{t}\right)\right)$ holds a.e. in $(0, T) \times \Omega$; hence, $\boldsymbol{u}$ solves the original problem (1-1).

We start with estimates uniform with respect to $\lambda$. In the following the positive constant $C$ is always independent of $\lambda$ but it can depend on the data $\left(\boldsymbol{f}, \rho_{0}, \boldsymbol{u}_{0}, \boldsymbol{v}_{0}, p, r, v_{0}, v_{1}, v_{2}\right)$. From Lemma 3.1 we know that

$$
\left\|\nabla^{2} \boldsymbol{u}\right\|_{L^{\infty}\left(0, T ; L^{2}\right)}+\left\|\boldsymbol{u}_{t t}\right\|_{L^{\infty}\left(0, T ; L^{2}\right)} \leq C\left(1+\left\|\mathbf{G}^{\lambda}\left(\mathbf{D}\left(\boldsymbol{v}_{0}\right)\right)\right\|_{1,2}\right) .
$$

This estimate is still $\lambda$-dependent. However, using the definition of $\mathbf{G}^{\lambda}$ and the assumptions on the data (1-15), we see that

$$
\left\|\mathbf{G}^{\lambda}\left(\mathbf{D}\left(\boldsymbol{v}_{0}\right)\right)\right\|_{1,2} \leq C\left(1+\left\|\left|\mathbf{D}\left(\boldsymbol{v}_{0}\right)\right|^{r-1}\right\|_{2}+\left\|\left|\mathbf{D}\left(\boldsymbol{v}_{0}\right)\right|^{r-2}\left|\nabla^{2} \boldsymbol{v}_{0}\right|\right\|_{2}\right) \leq C\left(1+\left\|\boldsymbol{v}_{0}\right\|_{2, p}^{r-1}\right),
$$

where for the last inequality we used the Hölder inequality and the embedding $W^{2, p} \hookrightarrow W^{1, \infty}$ (valid for $p>2$ ). Consequently, we see that (4-15) can be rewritten as

$$
\left\|\nabla^{2} \boldsymbol{u}\right\|_{L^{\infty}\left(0, T ; L^{2}\right)}+\left\|\boldsymbol{u}_{t t}\right\|_{L^{\infty}\left(0, T ; L^{2}\right)} \leq C .
$$

Uniform estimates on $\nabla^{2} \boldsymbol{u}_{t}$ are obtained by the same method as in the proof of Lemma 3.3. We rewrite (4-9) for a.e. $t \in(0, T)$ as

$$
-\operatorname{div} \mathbf{G}^{\lambda}\left(\mathbf{D}\left(\boldsymbol{u}_{t}(t)\right)\right)=\rho_{0} \boldsymbol{f}(t)+\operatorname{div} \mathbf{H}(\mathbf{D}(\boldsymbol{u}(t)))-\rho_{0} \boldsymbol{u}_{t t}(t) .
$$

This equation holds pointwise in $\Omega$ due to (4-11) and it is allowed to test it with $\boldsymbol{u}_{t}(t)$ and $-\Delta \boldsymbol{u}_{t}(t)$. Doing so, one gets with help of (4-16) and (4-8) that

$$
\int_{\Omega}\left(1+\bar{\lambda}\left(\mathbf{D}\left(\boldsymbol{u}_{t}(t)\right)\right)^{2}\right)^{(r-2) / 2}\left|\nabla^{2} \boldsymbol{u}_{t}(t)\right|^{2} \leq C \quad \text { for any } t \in(0, T),
$$


and by a simple algebraic manipulation we deduce that

$$
\left\|\left(1+\bar{\lambda}\left(\mathbf{D}\left(\boldsymbol{u}_{t}\right)\right)^{2}\right)^{r / 4}\right\|_{L^{\infty}\left(0, T ; W^{1,2}\right)} \leq C .
$$

Finally, we combine the nonuniform estimate (4-13) with the uniform ones (4-16) and (4-17) to deduce (4-14). First, we consider $\bar{s} \in(2, s)$ and $\alpha \in(0,2)$ such that $1=\alpha / 2+(\bar{s}-\alpha) / s$, i.e.,

$$
\bar{s}-2=(2-\alpha)(s-2) / 2 .
$$

We use the Hölder inequality to get

$$
\begin{aligned}
& \left\|\left(1+\bar{\lambda}\left(\mathbf{D}\left(\boldsymbol{u}_{t}\right)\right)^{2}\right)^{r / 4}\right\|_{L^{\infty}\left(0, T ; W^{1, \bar{s}}\right)}^{\bar{s}} \\
& \quad \leq C\left\|\left(1+\bar{\lambda}\left(\mathbf{D}\left(\boldsymbol{u}_{t}\right)\right)^{2}\right)^{r / 4}\right\|_{L^{\infty}\left(0, T ; W^{1,2}\right)}^{\alpha}\left\|\left(1+\bar{\lambda}\left(\mathbf{D}\left(\boldsymbol{u}_{t}\right)\right)^{2}\right)^{r / 4}\right\|_{L^{\infty}\left(0, T ; W^{1, s}\right)}^{\bar{s}-\alpha} .
\end{aligned}
$$

To estimate the term on the right, we use the definition of $\bar{\lambda}$, the uniform estimate (4-17) and the nonuniform estimate (4-13) to conclude that

$$
\begin{aligned}
\left\|\left(1+\bar{\lambda}\left(\mathbf{D}\left(\boldsymbol{u}_{t}\right)\right)^{2}\right)^{r / 4}\right\|_{L^{\infty}\left(0, T ; W^{1, \bar{s}}\right)}^{\bar{s}} & \leq C\left(1+\left\|\nabla\left(1+\bar{\lambda}\left(\mathbf{D}\left(\boldsymbol{u}_{t}\right)\right)^{2}\right)^{r / 4}\right\|_{L^{\infty}\left(0, T ; L^{s}\right)}^{\bar{s}-\alpha}\right) \\
& \leq C\left(1+\left\|\nabla^{2} \boldsymbol{u}_{t}\right\|_{L^{\infty}\left(0, T ; L^{s}\right)}^{\bar{s}-\alpha} \lambda^{(r-2)(\bar{s}-\alpha) / 2}\right) \\
& \leq C \lambda^{(r-2)(\bar{s}-\alpha)(3 / 2)} .
\end{aligned}
$$

Finally, we focus on finding such $\lambda>\lambda_{0}$ so that (4-14) holds. Using the embedding theorem $W^{1, \bar{s}} \hookrightarrow L^{\infty}$ with the precise embedding constant (see [Ziemer 1989, proof of Theorem 2.4.1]), the definition (4-14) of $M$ and the estimate (4-19), we get

$$
\begin{aligned}
M^{r / 4} & \leq\left(\frac{C}{\bar{s}-2}\right)^{1-1 / \bar{s}}\left\|\left(1+\bar{\lambda}\left(\mathbf{D}\left(\boldsymbol{u}_{t}\right)\right)^{2}\right)^{r / 4}\right\|_{L^{\infty}\left(0, T ; W^{1, \bar{s}}\right)} \\
& \leq\left(\frac{C}{\bar{s}-2}\right)^{1-1 / \bar{s}} \lambda^{\frac{3(r-2)(\bar{s}-\alpha)}{2 \bar{s}}} .
\end{aligned}
$$

Hence, to show (4-14) and consequently to finish the proof, it is enough to find $\lambda>\lambda_{0}, \bar{s} \in(2, s)$ and $\alpha \in(0,2)$ fulfilling (4-18) such that

$$
\left(\frac{C}{\bar{s}-2}\right)^{1-1 / \bar{s}} \lambda^{\frac{3(r-2)(\bar{s}-\alpha)}{2 \bar{s}}} \leq \lambda^{r / 2} .
$$

Next, using (4-12) and (4-18) it is not difficult to deduce the identities

$$
\begin{aligned}
\left(\frac{C}{\bar{s}-2}\right)^{1-1 / \bar{s}} \lambda^{(r-2)(3 / 2) \frac{\bar{s}-\alpha}{\bar{s}}} & =\left(\frac{2 C}{(2-\alpha)(s-2)}\right)^{1-1 / \bar{s}} \lambda^{(r-2)(3 / 2) \frac{\bar{s}-\alpha}{\bar{s}}} \\
& =\left(\frac{2 C}{(2-\alpha) R}\right)^{1-1 / \bar{s}} \lambda^{(r-2)\left(1-\frac{1}{\bar{s}}+(3 / 2) \frac{\bar{s}-\alpha}{\bar{s}}\right)}
\end{aligned}
$$


and we see that (4-21) is equivalent to

$$
\left(\frac{2 C}{(2-\alpha) R}\right)^{1-1 / \bar{s}} \lambda^{(r-2)\left(1-\frac{1}{s}+\frac{\bar{s}-\alpha}{\bar{s}} \frac{3}{2}\right)} \leq \lambda^{r / 2} .
$$

Since $\lim _{\alpha \rightarrow 2-} \bar{s}=2$ we have

$$
\lim _{\alpha \rightarrow 2-}(r-2)\left(1-\frac{1}{\bar{s}}+\frac{\bar{s}-\alpha}{\bar{s}} \frac{3}{2}\right)=\frac{r-2}{2}<\frac{r}{2}
$$

and therefore it is always possible to find $\alpha \in(0,2)$ (and consequently $\bar{s}$ ) and $\varepsilon>0$ such that

$$
\frac{r}{2}-(r-2)\left(1-\frac{1}{\bar{s}}+\frac{\bar{s}-\alpha}{\bar{s}} \frac{3}{2}\right)>\epsilon .
$$

Thus, we fix such $\alpha$ and $\bar{s}$ and we see that to fulfill (4-22) it is enough to find $\lambda>\lambda_{0}$ such that

$$
\left(\frac{2 C}{(2-\alpha) R}\right)^{1-1 / \bar{s}} \leq \lambda^{\varepsilon}
$$

which is clearly possible and therefore the proof of (1-17) for the case $r>2$ is complete. The regularity statement in (1-18) follows from Lemma A.1, part 2. Theorem 1.2 is proved.

\section{Proof of Theorem 1.3}

We start this section by formulating a result on $L^{p}$ regularity for certain parabolic systems with Hölder continuous coefficients.

Theorem 5.1. Let $d \in \mathbb{N}, \alpha \in(0,1], p>1, \Omega=(0,1)^{d}$ and $Q=(0, T) \times \Omega$. Assume that $\mathbf{A}_{i j}^{k l}:(0, T) \times \mathbb{R}^{d} \rightarrow \mathbb{R}$ satisfy the symmetry condition (2-1) and in addition for all $i, j, k, l \in\{1, \ldots, d\}$ there hold

$$
\begin{aligned}
& \mathbf{A}_{i j}^{k l} \in C^{0, \alpha}\left([0, T] \times \mathbb{R}^{d}\right), \quad \mathbf{A}_{i j}^{k l} \text { is periodic with respect to } \Omega, \\
& \exists \gamma>0, \forall \xi \in \mathbb{R}^{d \times d}, t>0, x \in \mathbb{R}^{d}: \quad(\mathbf{A}(t, x): \xi \otimes \xi) \geq \gamma\left|\boldsymbol{\xi}_{\mathrm{sym}}\right|^{2} .
\end{aligned}
$$

Let $1<q<p$ and $\boldsymbol{w} \in L^{q}\left(0, T, W^{2, q}\left(\mathbb{R}^{d}\right)\right)$ with $\partial_{t} \boldsymbol{w} \in L^{q}\left(0, T, L^{q}\left(\mathbb{R}^{d}\right)\right)$ be a strong solution of the problem

$$
\partial_{t} \boldsymbol{w}_{j}-\mathbf{A}_{i j}^{k l} \partial_{i} \partial_{k} \boldsymbol{w}_{l}=\mathbf{F}_{j} \quad \text { in }(0, T) \times \mathbb{R}^{d}
$$

such that $\boldsymbol{w}$ is periodic with respect to $\Omega$ and $\boldsymbol{w}(0, \cdot)=\boldsymbol{w}_{0}$, where $\boldsymbol{w}_{0} \in W_{\text {per }}^{2, p}(\Omega)$ and $\mathbf{F} \in L^{p}(Q)^{d}$. Then this solution satisfies $\nabla^{2} \boldsymbol{w} \in L^{p}(Q)^{d \times d \times d}, \partial_{t} \boldsymbol{w} \in L^{p}(Q)^{d}$ with the following uniform estimate:

$$
\exists C>0, \forall t \in(0, T): \quad\left\|\nabla^{2} \boldsymbol{w}\right\|_{p, Q_{t}}+\left\|\partial_{t} \boldsymbol{w}\right\|_{p, Q_{t}} \leq C\left(\|\mathbf{F}\|_{p, Q_{t}}+\left\|\boldsymbol{w}_{0}\right\|_{2, p}\right),
$$

where $Q_{t}=(0, t) \times \Omega$ and $C>0$ may depend on $T$ but is independent of $t$. 
Proof. First one finds a smooth approximation of the solution $\boldsymbol{w}$ by convolutions. Then it is possible to apply slightly modified $L^{p}$ theory from [Schlag 1996] provided $q>2$. If $q<2$ first one needs to develop by a duality argument an $L^{p}$ theory for $p<2$ based on the results from the same paper. (The result also follows from [Ladyzhenskaja et al. 1968, Theorem VII.10.4].)

In the rest of this section we provide only formal a priori estimates. However, they can be made rigorous by the method of Section 3, see the approximation (3-1) and the proof of Theorem 3.1.

Let $\boldsymbol{u}$ be the unique solution constructed in Theorem 1.2 and assume that it is sufficiently smooth. We suppose that all assumptions of Theorem 1.2 hold and show an estimate leading to (1-19). We denote $\boldsymbol{w}=\boldsymbol{u}_{t}$. It follows from Theorem 1.2 that $\boldsymbol{w}$ is a strong solution of the problem (5-3) with $\boldsymbol{w}_{0}=\boldsymbol{v}_{0}$ and

$$
\mathbf{A}_{i j}^{k l}=\frac{1}{\rho_{0}} \frac{\partial \mathbf{G}_{i j}\left(\mathbf{D}\left(\boldsymbol{u}_{t}\right)\right)}{\partial \mathbf{D}_{k l}}, \quad \mathbf{F}_{j}=\boldsymbol{f}_{j}+\frac{1}{\rho_{0}} \sum_{i=1}^{2} \partial_{\mathbf{D}} \mathbf{H}_{i j}(\mathbf{D} \boldsymbol{u}): \mathbf{D} \partial_{i} \boldsymbol{u} .
$$

Here, the symmetry of $\mathbf{A}$ was used. Since we already have (1-18) we know that $\mathbf{A}$ satisfies (5-1) and (5-2), $\partial_{\mathbf{D}} \mathbf{H}(\mathbf{D} \boldsymbol{u})$ is bounded and we can apply Theorem 5.1 to get

$$
\left\|\nabla^{2} \boldsymbol{u}_{t}\right\|_{p, Q_{t}}^{p}+\left\|\boldsymbol{u}_{t t}\right\|_{p, Q_{t}}^{p} \leq C\left(\|\boldsymbol{f}\|_{p, Q_{t}}^{p}+\left\|\nabla^{2} \boldsymbol{u}\right\|_{p, Q_{t}}^{p}+\left\|\boldsymbol{v}_{0}\right\|_{2, p}^{p}\right) .
$$

Using the inequality $\left\|\nabla^{2} \boldsymbol{u}(t)\right\|_{p}^{p} \leq C\left(\left\|\nabla^{2} \boldsymbol{u}_{0}\right\|_{p}^{p}+\left\|\nabla^{2} \boldsymbol{u}_{t}\right\|_{p, Q_{t}}^{p}+\left\|\nabla^{2} \boldsymbol{u}\right\|_{p, Q_{t}}^{p}\right)$ we conclude that

$$
\left\|\nabla^{2} \boldsymbol{u}(t)\right\|_{p}^{p} \leq C\left(\left\|\boldsymbol{u}_{0}\right\|_{2, p}^{p}+\left\|\boldsymbol{v}_{0}\right\|_{2, p}^{p}+\|\boldsymbol{f}\|_{p, Q_{T}}^{p}+\left\|\nabla^{2} \boldsymbol{u}\right\|_{p, Q_{t}}^{p}\right) .
$$

Gronwall's lemma with (5-4) then gives (1-19).

To get estimates for (1-21) we proceed similarly as in the first step. We a priori assume sufficient smoothness of $\boldsymbol{u}$ and define $\boldsymbol{w}=\partial_{k} \boldsymbol{u}_{t}$ for fixed $k \in\{1,2\}$. We differentiate (1-1) with respect to $x_{k}$ and find that $\boldsymbol{w}$ solves the problem (5-3) with $\boldsymbol{w}_{0}=\partial_{k} \boldsymbol{v}_{0}$ and

$$
\begin{aligned}
\mathbf{A}_{i j}^{k l}= & \frac{1}{\rho_{0}} \frac{\mathbf{G}_{i j}\left(\mathbf{D}\left(\boldsymbol{u}_{t}\right)\right)}{\partial \mathbf{D}_{k l}}, \\
\mathbf{F}_{j}= & \frac{1}{\rho_{0}}\left(\sum _ { i = 1 } ^ { 2 } \left[\partial_{\mathbf{D}}^{2} \mathbf{G}_{i j}\left(\mathbf{D}\left(\boldsymbol{u}_{t}\right)\right):\left(\mathbf{D}\left(\partial_{i} \boldsymbol{u}_{t}\right) \otimes \mathbf{D}\left(\partial_{k} \boldsymbol{u}_{t}\right)\right)+\partial_{\mathbf{D}} \mathbf{H}_{i j}(\mathbf{D}(\boldsymbol{u})): \mathbf{D}\left(\partial_{i} \partial_{k} \boldsymbol{u}\right)\right.\right. \\
& \left.\left.\quad+\partial_{\mathbf{D}}^{2} \mathbf{H}_{i j}(\mathbf{D}(\boldsymbol{u})):\left(\mathbf{D}\left(\partial_{i} \boldsymbol{u}\right) \otimes \mathbf{D}\left(\partial_{k} \boldsymbol{u}\right)\right)\right]-\partial_{k} \rho_{0} \partial_{t}^{2} \boldsymbol{u}_{j}+\partial_{k}\left(\rho_{0} \boldsymbol{f}_{j}\right)\right) .
\end{aligned}
$$

For an arbitrary $\sigma \in(1, p], t \in(0, T)$ we obtain from the properties of $\mathbf{G}, \mathbf{H}$ and (1-18) that 


$$
\|\mathbf{F}\|_{\sigma, Q_{t}} \leq C\left(1+\left\|\nabla^{2} \boldsymbol{u}_{t}\right\|_{2 \sigma, Q_{t}}^{2}+\left\|\nabla^{3} \boldsymbol{u}\right\|_{\sigma, Q_{t}}+\|\nabla \boldsymbol{u}\|_{2 \sigma, Q_{t}}^{2}+\left\|\boldsymbol{u}_{t t}\right\|_{\sigma, Q_{t}}\right) .
$$

The constant $C>0$ may depend on $\mathbf{H}, \mathbf{G}, T, \boldsymbol{u}_{0}, \rho_{0}$.

Using Theorem 5.1 we obtain, since $k=1,2$ was arbitrary,

$$
\begin{aligned}
& \left\|\nabla \boldsymbol{u}_{t t}\right\|_{\sigma, Q_{t}}+\left\|\nabla^{3} \boldsymbol{u}_{t}\right\|_{\sigma, Q_{t}} \\
& \leq C\left(1+\left\|\nabla^{2} \boldsymbol{u}_{t}\right\|_{2 \sigma, Q_{t}}^{2}+\left\|\nabla^{3} \boldsymbol{u}\right\|_{\sigma, Q_{t}}+\|\nabla \boldsymbol{u}\|_{2 \sigma, Q_{t}}^{2}+\left\|\boldsymbol{u}_{t t}\right\|_{\sigma, Q_{t}}+\left\|\boldsymbol{v}_{0}\right\|_{3, \sigma}\right) .
\end{aligned}
$$

Now we use the inequality $\left\|\nabla^{3} \boldsymbol{u}(t)\right\|_{\sigma}^{\sigma} \leq C\left(\left\|\nabla^{3} \boldsymbol{u}\right\|_{\sigma, Q_{t}}^{\sigma}+\left\|\nabla^{3} \boldsymbol{u}_{t}\right\|_{\sigma, Q_{t}}^{\sigma}+\left\|\nabla^{3} \boldsymbol{u}_{0}\right\|_{\sigma}^{\sigma}\right)$ to get

$$
\begin{aligned}
& \left\|\nabla^{3} \boldsymbol{u}(t)\right\|_{\sigma, \Omega}^{\sigma}+\left\|\nabla \boldsymbol{u}_{t t}\right\|_{\sigma, Q_{t}}^{\sigma}+\left\|\nabla^{3} \boldsymbol{u}_{t}\right\|_{\sigma, Q_{t}}^{\sigma} \\
& \quad \leq C\left(1+\left\|\nabla^{2} \boldsymbol{u}_{t}\right\|_{2 \sigma, Q_{t}}^{2 \sigma}+\left\|\nabla^{3} \boldsymbol{u}\right\|_{\sigma, Q_{t}}^{\sigma}+\|\nabla \boldsymbol{u}\|_{2 \sigma, Q_{t}}^{2 \sigma}+\left\|\boldsymbol{u}_{t t}\right\|_{\sigma, Q_{t}}^{\sigma}+\left\|\boldsymbol{u}_{0}\right\|_{3, \sigma}^{\sigma}+\left\|\boldsymbol{v}_{0}\right\|_{3, \sigma}^{\sigma}\right) .
\end{aligned}
$$

Due to the assumption on $\boldsymbol{u}_{0}$ and $\boldsymbol{v}_{0}$ we know that $\left\|\boldsymbol{u}_{0}\right\|_{3, \sigma}^{\sigma}+\left\|\boldsymbol{v}_{0}\right\|_{3, \sigma}^{\sigma}<+\infty$ for all $\sigma \leq p$. If $\left\|\nabla^{2} \boldsymbol{u}_{t}\right\|_{2 \sigma, Q_{t}}^{2 \sigma}+\|\nabla \boldsymbol{u}\|_{2 \sigma, Q_{t}}^{2 \sigma}+\left\|\boldsymbol{u}_{t t}\right\|_{\sigma, Q_{t}}^{\sigma}$ is bounded we get by Gronwall's inequality

$$
\left\|\nabla \boldsymbol{u}_{t t}\right\|_{\sigma, Q_{t}}^{\sigma}+\left\|\nabla^{3} \boldsymbol{u}_{t}\right\|_{\sigma, Q_{t}}^{\sigma}<+\infty .
$$

This is always true for $\sigma \in(1, s / 2]$, where $s>2$ is taken from Theorem 1.2. By the following multiplicative inequality [Ladyzhenskaja et al. 1968, Theorem II.2.2] we get that, for any measurable function $z$,

$$
\|z\|_{\sigma(s+2) / 2, Q}^{\sigma(s+2) / 2} \leq C\|z\|_{L^{\infty}\left(0, T ; L^{s}\right)}^{\sigma s / 2}\|\nabla z\|_{\sigma, Q}^{\sigma} .
$$

If we take into account (5-5) and (1-17) we find that, for $\sigma \in(1, s / 2]$,

$$
\left\|\nabla^{2} \boldsymbol{u}\right\|_{\sigma(s+2) / 2}+\left\|\nabla^{2} \boldsymbol{u}_{t}\right\|_{\sigma(s+2) / 2}+\left\|\boldsymbol{u}_{t t}\right\|_{\sigma(s+2) / 2, Q}<+\infty .
$$

Since $\sigma \frac{s+2}{2}>2 \sigma$ for all $\sigma>1$ we get the statement (1-21) of Theorem 1.3 after finite number of iterations.

\section{Appendix: Proof of Lemma 2.1}

First, we sketch the proof of Lemma 2.1. We focus on main differences compared to [Nečas and Šverák 1991], where the full gradient case is treated.

Proof of Lemma 2.1. The existence and uniqueness of a weak solution to (2-3) is standard. Therefore, we focus here only on the proof of (2-5). We provide only the formal proof but everything can be done rigorously by mollifying $A$ and $\boldsymbol{w}_{0}$, applying standard results for the heat equation, deriving uniform bounds of the type (2-5) and then passing to the limit.

The main idea of the proof is to use $\boldsymbol{w}|\boldsymbol{w}|^{s-2}$ as a test function in the weak formulation of (2-3). Due to the presence of a nonlinearity in the test function we 
obtain a pollution term coming from the elliptic term. To handle it, we use a simple inequality that can be deduced by an integration by parts formula. Hence, for any $s \geq 2$ and any smooth periodic $\boldsymbol{u}$ we have

$$
\begin{aligned}
& \int_{\Omega}|\boldsymbol{u}|^{s-2}|\nabla \boldsymbol{u}|^{2} \\
& =-\int_{\Omega}|\boldsymbol{u}|^{s-2} \triangle \boldsymbol{u} \cdot \boldsymbol{u}-(s-2) \int_{\Omega}|\boldsymbol{u}|^{s-2}|\nabla| \boldsymbol{u}||^{2} \\
& =-\left.(s-2) \int_{\Omega}|\boldsymbol{u}|^{s-2}|\nabla| \boldsymbol{u}\right|^{2}-2 \int_{\Omega}|\boldsymbol{u}|^{s-2} \operatorname{div}(\mathbf{D}(\boldsymbol{u})) \cdot \boldsymbol{u}+\int_{\Omega}|\boldsymbol{u}|^{s-2} \nabla(\operatorname{div} \boldsymbol{u}) \cdot \boldsymbol{u} \\
& =-(s-2) \int_{\Omega}|\boldsymbol{u}|^{s-2}|\nabla| \boldsymbol{u}||^{2}+2 \int_{\Omega}|\boldsymbol{u}|^{s-2}|\mathbf{D}(\boldsymbol{u})|^{2}+2(s-2) \int_{\Omega}|\boldsymbol{u}|^{s-3} \mathbf{D}(\boldsymbol{u}) \boldsymbol{u} \cdot \nabla|\boldsymbol{u}| \\
& \quad-\int_{\Omega}|\boldsymbol{u}|^{s-2}|\operatorname{div} \boldsymbol{u}|^{2}-(s-2) \int_{\Omega}|\boldsymbol{u}|^{s-3} \operatorname{div} \boldsymbol{u} \nabla|\boldsymbol{u}| \cdot \boldsymbol{u} .
\end{aligned}
$$

Consequently, moving the term with the good sign to the left side we obtain the inequality

$$
\begin{array}{r}
\int_{\Omega}\left(|\boldsymbol{u}|^{s-2}|\operatorname{div} \boldsymbol{u}|^{2}+(s-2)|\boldsymbol{u}|^{s-2}|\nabla| \boldsymbol{u}||^{2}+|\boldsymbol{u}|^{s-2}|\nabla \boldsymbol{u}|^{2}\right) \\
\leq 2 \int_{\Omega}|\boldsymbol{u}|^{s-2}|\mathbf{D}(\boldsymbol{u})|^{2}+2(s-2) \int_{\Omega}|\boldsymbol{u}|^{s-2}|\mathbf{D}(\boldsymbol{u})||\nabla| \boldsymbol{u}|| \\
+(s-2) \int_{\Omega}|\boldsymbol{u}|^{s-2}|\operatorname{div} \boldsymbol{u}||\nabla| \boldsymbol{u}|| .
\end{array}
$$

Finally, using the pointwise estimate $|\operatorname{div} \boldsymbol{u}| \leq C|\mathbf{D}(\boldsymbol{u})|$ and applying Young's inequality we deduce that for any $s>2$ there exists $C_{s}>0$ such that

$$
\int_{\Omega}|\boldsymbol{u}|^{s-2}|\nabla \boldsymbol{u}|^{2} \leq C_{s} \int_{\Omega}|\boldsymbol{u}|^{s-2}|\mathbf{D}(\boldsymbol{u})|^{2} .
$$

If we restrict to $s \in[2,10]$ we can find $C^{*}>0$ such that for all $s \in[2,10], C_{s}<C^{*}$.

With estimate (A-1) we can easily continue by using the standard procedure; see [Nečas and Šverák 1991; Frehse and Seregin 1999]. We test (2-3) by $|\boldsymbol{w}|^{s-2} \boldsymbol{w}$ with arbitrary $s \in[2,4]$ to get

$$
\text { (A-2) } \begin{aligned}
\frac{1}{s} \frac{d}{d t}\|\boldsymbol{w}\|_{s}^{s}+\int_{\Omega}|\boldsymbol{w}|^{s-2} \mathbf{A D}(\boldsymbol{w}) \cdot \mathbf{D}(\boldsymbol{w}) \\
=-\int_{\Omega} \mathbf{F} \cdot \nabla\left(|\boldsymbol{w}|^{s-2} \boldsymbol{w}\right)-\int_{\Omega} \mathbf{A D}(\boldsymbol{w}) \cdot\left(\nabla|\boldsymbol{w}|^{s-2} \otimes \boldsymbol{w}\right) .
\end{aligned}
$$

Consequently, using (2-2) and (A-1) we observe that (we use Young's inequality to 
get the second estimate)

$$
\text { (A-3) } \begin{aligned}
\frac{1}{s} \frac{d}{d t}\|\boldsymbol{w}\|_{s}^{s} & +\lambda_{1} \int_{\Omega}|\boldsymbol{w}|^{s-2}|\mathbf{D}(\boldsymbol{w})|^{2} \\
& \leq C\left(\int_{\Omega}|\mathbf{F}||\boldsymbol{w}|^{s-2}|\nabla \boldsymbol{w}|+\lambda_{2}(s-2) \int_{\Omega}|\boldsymbol{w}|^{s-2}|\nabla \boldsymbol{w}|^{2}\right) \\
& \leq\left(C \lambda_{2}(s-2)+\frac{\lambda_{1}}{2 C^{*}}\right) \int_{\Omega}|\boldsymbol{w}|^{s-2}|\nabla \boldsymbol{w}|^{2}+\frac{C^{2} C^{*}}{2 \lambda_{1}} \int_{\Omega}|\mathbf{F}|^{2}|\boldsymbol{w}|^{s-2} \\
& \leq \lambda_{1} \int_{\Omega}|\boldsymbol{w}|^{s-2}|\mathbf{D}(\boldsymbol{w})|^{2}+\frac{C^{2} C^{*}}{2 \lambda_{1}} \int_{\Omega}|\mathbf{F}|^{2}|\boldsymbol{w}|^{s-2},
\end{aligned}
$$

provided that

$$
C C^{*} \lambda_{2}(s-2)+\lambda_{1} / 2<\lambda_{1} .
$$

Thus, defining $L:=1 /\left(2 C C^{*}\right)$, we see that for all $2 \leq s \leq 2+L \lambda_{1} / \lambda_{2}$ the condition (A-4) is automatically met and the inequality (A-3) implies that

$$
\frac{d}{d t}\|\boldsymbol{w}\|_{s}^{s} \leq \frac{C}{\lambda_{1}} \int_{\Omega}|\mathbf{F}|^{2}|\boldsymbol{w}|^{s-2} \leq \frac{C}{\lambda_{1}}\|\mathbf{F}\|_{s}^{2}\|\boldsymbol{w}\|_{s}^{s-2}
$$

and we finally obtain

$$
\frac{d}{d t}\|\boldsymbol{w}\|_{s}^{2} \leq \frac{C}{\lambda_{1}}\|\mathbf{F}\|_{s}^{2},
$$

which leads to (2-5) after integration with respect to $t \in(0, T)$.

Lemma A.1 (See also [Ladyzhenskaja et al. 1968]). Let $T>0$ and $\Omega \subset \mathbb{R}^{2}$. Assume that $p>4$; then the embedding

$$
W^{1, p}\left(0, T ; L^{p}\right) \cap L^{p}\left(0, T ; W_{\text {per }}^{2, p}\right) \hookrightarrow \mathscr{C}\left([0, T], \mathscr{C}_{\text {per }}^{1}(\bar{\Omega})\right) .
$$

holds. In addition for any $s>2$ and $\alpha \in(0,(1-2 / s) / 3)$ we have

$$
W^{1, \infty}\left(0, T ; L^{s}\right) \cap L^{\infty}\left(0, T ; W^{2, s}\right) \hookrightarrow \mathscr{C}^{0, \alpha}\left([0, T], \mathscr{C}_{\text {per }}^{1, \alpha}(\bar{\Omega})\right) .
$$

Proof. By using an interpolation theorem (see [Amann 2000, proof of Corollary 4.5(ii)]) we find that, for any $\alpha \in[0,1], p_{1}, p_{2} \in(1, \infty)$,

$$
W^{1, p_{1}}\left(0, T ; L^{p_{2}}\right) \cap L^{p_{1}}\left(0, T ; W_{\mathrm{per}}^{2, p_{2}}\right) \hookrightarrow W^{\alpha, p_{1}}\left(0, T ; W_{\mathrm{per}}^{2(1-\alpha), p_{2}}\right) .
$$

Consequently, setting $\alpha:=\frac{1}{4}, p_{1}=p_{2}:=p$ and using the standard Sobolev embedding we get (A-5), provided that $p>4$.

To prove the second part of the lemma, we first note that for any $p \in[1, \infty]$ we have $W^{1, \infty}\left(0, T ; L^{s}\right) \cap L^{\infty}\left(0, T ; W^{2, s}\right) \hookrightarrow W^{1, p}\left(0, T ; L^{s}\right) \cap L^{p}\left(0, T ; W^{2, s}\right)$. Consequently, setting $p_{2}:=s, p_{1}:=p$ in (A-7), we deduce that

$$
W^{1, \infty}\left(0, T ; L^{s}\right) \cap L^{\infty}\left(0, T ; W_{\text {per }}^{2, s}\right) \hookrightarrow W^{\alpha, p}\left(0, T ; W_{\text {per }}^{2(1-\alpha), s}\right)
$$


for any $p \in(1, \infty)$ and any $\alpha \in[0,1]$. Finally, assuming that $p>\frac{2 s}{s-2}$ and setting

$$
\alpha:=\frac{p s-2 p+s}{3 p s}
$$

in (A-8), we get after using the standard embedding theorem that

$$
W^{1, \infty}\left(0, T ; L^{s}\right) \cap L^{\infty}\left(0, T ; W_{\mathrm{per}}^{2, s}\right) \hookrightarrow \mathscr{C}^{0, \beta}\left(0, T ; \mathscr{C}_{\mathrm{per}}^{1, \beta}(\bar{\Omega})\right)
$$

with $\beta:=\frac{1}{3}-\frac{2}{3 s}-\frac{2}{3 p}$. Since $p$ is arbitrarily large the embedding (A-6) follows.

\section{References}

[Amann 2000] H. Amann, "Compact embeddings of vector-valued Sobolev and Besov spaces", Glas. Mat. Ser. III 35:1 (2000), 161-177. MR 2001h:46056 Zbl 0997.46029

[Boyarskiı̆ 1957] B. V. Boyarskiı̆, "Generalized solutions of a system of differential equations of first order and of elliptic type with discontinuous coefficients", Mat. Sb. N.S. 43(85) (1957), 451-503. In Russian. MR 21 \#5058

[Bulíček et al. 2012] M. Bulíček, J. Málek, and K. R. Rajagopal, "On Kelvin-Voigt model and its generalizations", Evolution Equations and Control Theory 1:1 (2012), 17-42.

[Demoulini 2000] S. Demoulini, "Weak solutions for a class of nonlinear systems of viscoelasticity", Arch. Ration. Mech. Anal. 155:4 (2000), 299-334. MR 2002d:74027 Zbl 0991.74021

[DiBenedetto and Friedman 1984] E. DiBenedetto and A. Friedman, "Regularity of solutions of nonlinear degenerate parabolic systems", J. Reine Angew. Math. 349 (1984), 83-128. MR 85j:35089 Zbl 0527.35038

[DiBenedetto and Friedman 1985] E. DiBenedetto and A. Friedman, "Hölder estimates for nonlinear degenerate parabolic systems”, J. Reine Angew. Math. 357 (1985), 1-22. MR 87f:35134a Zbl 0549.35061

[Feireisl and Novotný 2009] E. Feireisl and A. Novotný, Singular limits in thermodynamics of viscous fluids, Birkhäuser, Basel, 2009. MR 2011b:35001 Zbl 1176.35126

[Frehse and Seregin 1999] J. Frehse and G. A. Seregin, "Full regularity for a class of degenerated parabolic systems in two spatial variables", Manuscripta Math. 99:4 (1999), 517-539. MR 2000e:35117 Zbl 0931.35029

[Friedman and Nečas 1988] A. Friedman and J. Nečas, "Systems of nonlinear wave equations with nonlinear viscosity", Pacific J. Math. 135:1 (1988), 29-55. MR 90b:35152 Zbl 0685.35070

[Fung 1993] Y.-C. Fung, Biomechanics: Mechanical properties of living tissues, 2nd ed., Springer, New York, 1993.

[Kaplický et al. 2002] P. Kaplický, J. Málek, and J. Stará, "Global-in-time Hölder continuity of the velocity gradients for fluids with shear-dependent viscosities", NoDEA Nonlinear Differential Equations Appl. 9:2 (2002), 175-195. MR 2003i:35233 Zbl 0991.35066

[Ladyzhenskaja et al. 1968] O. A. Ladyzhenskaja, V. A. Solonnikov, and N. N. Ural'ceva, Linear and quasilinear equations of parabolic type, vol. 23, Translations of Mathematical Monographs, American Mathematical Society, Providence, 1968. MR 39 \#3159b

[Meyers 1963] N. G. Meyers, "An $L^{p}$ e-estimate for the gradient of solutions of second order elliptic divergence equations", Ann. Scuola Norm. Sup. Pisa (3) 17 (1963), 189-206. MR 28 \#2328 Zbl 0127.31904 
[Nečas 1967] J. Nečas, "Sur la régularité des solutions variationelles des équations elliptiques nonlinéaires d'ordre $2 k$ en deux dimensions", Ann. Scuola Norm. Sup. Pisa (3) 21 (1967), 427-457. MR 37 \#2057 Zbl 0171.09401

[Nečas and Šverák 1991] J. Nečas and V. Šverák, "On regularity of solutions of nonlinear parabolic systems", Ann. Scuola Norm. Sup. Pisa Cl. Sci. (4) 18:1 (1991), 1-11. MR 92d:35058 Zbl 0735.35035

[Novotný and Straškraba 2004] A. Novotný and I. Straškraba, Introduction to the mathematical theory of compressible flow, Oxford Lecture Series in Mathematics and its Applications 27, Oxford University Press, 2004. MR 2005i:35220 Zbl 1088.35051

[Rajagopal 2009] K. R. Rajagopal, "A note on a reappraisal and generalization of the Kelvin-Voigt model”, Mech. Res. Comm. 36:2 (2009), 232-235.

[Ramberg and Osgood 1943] W. Ramberg and W. R. Osgood, "Description of stress-strain curves by three parameters", Tech. Notes Nat. Adv. Comm. Aeronaut. 1943:902 (1943), 1-13. MR 7,229h

[Schlag 1996] W. Schlag, "Schauder and $L^{p}$ estimates for parabolic systems via Campanato spaces", Comm. Partial Differential Equations 21:7-8 (1996), 1141-1175. MR 97k:35108 Zbl 0864.35023

[Thomson 1865] W. Thomson, "On the elasticity and viscosity of metals", Proc. Roy. Soc. London 14 (1865), 289-297.

[Tvedt 2008] B. Tvedt, "Quasilinear equations for viscoelasticity of strain-rate type", Arch. Ration. Mech. Anal. 189:2 (2008), 237-281. MR 2009m:74028 Zbl 1147.74008

[Voigt 1892] W. Voigt, "Ueber innere Reibung fester Körper, insbesondere der Metalle", Annalen der Physik 283:12 (1892), 671-693. JFM 24.0932.01

[Ziemer 1989] W. P. Ziemer, Weakly differentiable functions: Sobolev spaces and functions of bounded variation, Graduate Texts in Mathematics 120, Springer, New York, 1989. MR 91e:46046 Zbl 0692.46022

Received September 19, 2011. Revised November 8, 2012.

\section{MiroslaV BULÍČEK}

Mathematical Institute, Faculty of Mathematics and Physics

Charles University in Prague

18675 PRAHA 8

CZECH REPUBLIC

mbu18060@karlin.mff.cuni.cz

\section{PETR KAPLICKÝ}

DePartment of Mathematical Analysis, FaCUlty of Mathematics and Physics Charles University in PRAgue

18675 PRAHA 8

CZECH REPUBLIC

kaplicky@karlin.mff.cuni.cz

\section{MARK Steinhauer}

Mathematical Institute

UNIVERSITY OF KOBLENZ-LANDAU

CAMPUS KOBLENZ

56070 KOBLENZ

GERMANY

steinhauerm@uni-koblenz.de 


\title{
PACIFIC JOURNAL OF MATHEMATICS
}

\author{
msp.org/pjm
}

Founded in 1951 by E. F. Beckenbach (1906-1982) and F. Wolf (1904-1989)

\section{EDITORS}

V. S. Varadarajan (Managing Editor)

Department of Mathematics

University of California

Los Angeles, CA 90095-1555

pacific@math.ucla.edu

Paul Balmer

Department of Mathematics

University of California

Los Angeles, CA 90095-1555

balmer@math.ucla.edu

Daryl Cooper

Department of Mathematics

University of California

Santa Barbara, CA 93106-3080 cooper@math.ucsb.edu

Jiang-Hua $\mathrm{Lu}$

Department of Mathematics

The University of Hong Kong

Pokfulam Rd., Hong Kong jhlu@maths.hku.hk
Don Blasius

Department of Mathematics University of California

Los Angeles, CA 90095-1555

blasius@math.ucla.edu

Robert Finn

Department of Mathematics Stanford University

Stanford, CA 94305-2125

finn@math.stanford.edu

Sorin Popa

Department of Mathematics

University of California

Los Angeles, CA 90095-1555

popa@math.ucla.edu

Paul Yang

Department of Mathematics

Princeton University

Princeton NJ 08544-1000

yang@math.princeton.edu

\section{PRODUCTION}

Silvio Levy, Scientific Editor, production@msp.org

\section{SUPPORTING INSTITUTIONS}

ACADEMIA SINICA, TAIPEI

CALIFORNIA INST. OF TECHNOLOGY

INST. DE MATEMÁTICA PURA E APLICADA

KEIO UNIVERSITY

MATH. SCIENCES RESEARCH INSTITUTE

NEW MEXICO STATE UNIV.

OREGON STATE UNIV.

\author{
STANFORD UNIVERSITY \\ UNIV. OF BRITISH COLUMBIA \\ UNIV. OF CALIFORNIA, BERKELEY \\ UNIV. OF CALIFORNIA, DAVIS \\ UNIV. OF CALIFORNIA, LOS ANGELES \\ UNIV. OF CALIFORNIA, RIVERSIDE \\ UNIV. OF CALIFORNIA, SAN DIEGO \\ UNIV. OF CALIF., SANTA BARBARA
}

\author{
Vyjayanthi Chari \\ Department of Mathematics \\ University of California \\ Riverside, CA 92521-0135 \\ chari@math.ucr.edu \\ Kefeng Liu \\ Department of Mathematics \\ University of California \\ Los Angeles, CA 90095-1555 \\ liu@math.ucla.edu \\ Jie Qing \\ Department of Mathematics \\ University of California \\ Santa Cruz, CA 95064 \\ qing@cats.ucsc.edu
}

These supporting institutions contribute to the cost of publication of this Journal, but they are not owners or publishers and have no responsibility for its contents or policies.

See inside back cover or msp.org/pjm for submission instructions.

The subscription price for 2013 is US \$400/year for the electronic version, and \$485/year for print and electronic.

Subscriptions, requests for back issues and changes of subscribers address should be sent to Pacific Journal of Mathematics, P.O. Box 4163, Berkeley, CA 94704-0163, U.S.A. The Pacific Journal of Mathematics is indexed by Mathematical Reviews, Zentralblatt MATH, PASCAL CNRS Index, Referativnyi Zhurnal, Current Mathematical Publications and the Science Citation Index.

The Pacific Journal of Mathematics (ISSN 0030-8730) at the University of California, c/o Department of Mathematics, 798 Evans Hall \#3840, Berkeley, CA 94720-3840, is published monthly except July and August. Periodical rate postage paid at Berkeley, CA 94704, and additional mailing offices. POSTMASTER: send address changes to Pacific Journal of Mathematics, P.O. Box 4163, Berkeley, CA 94704-0163.

PJM peer review and production are managed by EditFLOW ${ }^{\circledR}$ from Mathematical Sciences Publishers.

PUBLISHED BY

mathematical sciences publishers

nonprofit scientific publishing

http://msp.org/

(C) 2013 Mathematical Sciences Publishers 


\section{PACIFIC JOURNAL OF MATHEMATICS}

Volume $262 \quad$ No. $1 \quad$ March 2013

On the second $K$-group of a rational function field

KARIM JOHANNES BECHER and MÉLANIE RACZEK

On existence of a classical solution to a generalized Kelvin-Voigt model

Miroslav BulíčEK, Petr KaPliCKÝ and Mark

STEINHAUER

A lower bound for eigenvalues of the poly-Laplacian with arbitrary order

Qing-Ming Cheng, Xuerong Qi and GuOXIN Wei

Quiver algebras, path coalgebras and coreflexivity

SORIN DĂSCĂLESCU, MIODRAG C. IOVANOV and CONSTANTIN

NĂSTĂSESCU

A positive density of fundamental discriminants with large regulator

ÉTIENNE FOUVRY and FLORENT JOUVE

On the isentropic compressible Euler equation with adiabatic index $\gamma=1$

Dong Li, ChANGXing MiaO and XiaOyi Zhang

Symmetric regularization, reduction and blow-up of the planar three-body problem

RICHARD MOECKEL and RICHARD MONTGOMERY

Canonical classes and the geography of nonminimal Lefschetz fibrations over $S^{2}$

YOSHIHISA SATO

Hilbert-Kunz invariants and Euler characteristic polynomials 